\title{
LA.SUB.- $93-210$
}

FINAL REPORT

\section{DISTRIBUTED DISCRETE EVENT SIMULATION}

\author{
Ronald C. De Vries \\ EECE Department \\ University of New Mexico \\ Albuquerque, New Mexico 87131 \\ Los Alamos National Laboratories \\ Contract No. BOA 9-X66-W2078-1 Task 12 \\ Bureau of Engineering Research \\ Report No. EECE-286(88) LANL-790-1
}

February, 1988

\section{DISCLAIMER}

This report was prepared as an account of work sponsored by an agency of the United States Government. Neither the United States Government nor any agency thereof, nor any of their employees, makes any warranty, express or implied, or assumes any legal liability or responsibility for the accuracy, completeness, or usefulness of any information, apparatus, product, or process disclosed, or represents that its use would not infringe privately owned rights. Reference herein to any specific commercial product, process, or service by trade name, trademark, manufacturer, or otherwise does not necessarily constitute or imply its endorsement, recommendation, or favoring by the United States Government or any agency thereof. The views and opinions of authors expressed herein do not necessarily state or reflect those of the United States Government or any agency thereof.

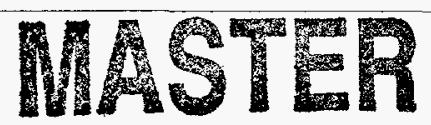




\section{Table of Contents}

\section{DISTRIBUTED DISCRETE EVENT SIMULATION}

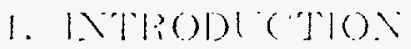

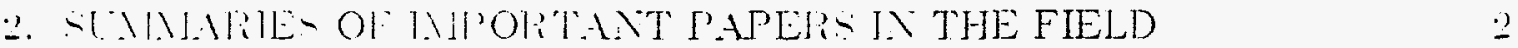

2.1 Methods Jurolving Local Clocks and Channel Clocks 2

2.1.1 Chandy and Misra. November, 1979

2.1.2 Chandy and Misra. September. 1979

2.2.3 Peacock. Nong. and Manning

2.1.4 Chandy and Misra. 1981

2.1.5 Smoliar

-.1.6 Yamamoto and Hasegawa

2.1.7 Chandy. Wisra. and Haas

2.1.8 Jeshida and Tokoro

2.1.9 Peed

2.1.10 Kinmar

2.1.11 Misra.

2.1.12 Bagrodia, Chandy, and Misra

2.2 Methods Involving Rollback

2.2.1 Jefferson

2.2.2 Berry and Jefferson

2.2.3 Sokol. Laskowsli, and Nugent

2.3 Methods Inrolving Both of the Previous Schemes

2.3.1 Clamance

2.4 Methods Involving Data Flow Concepts

3. CONCLUSIONS AND RESEARCH AREAS

3.1 Conclusion

42

3.2 Ideas for Future Research

4. REFERENCES 


\section{DISCLAMMER}

Portions of this document may be illegible in electronic image products. Images are produced from the best available original document. 


\section{DISTRIBUTED DISCRETE EVENT SIMULATION}

\section{INTRODUCTION}

The presentation given here is restricted to discrete event simulation. The complexity of and time required for many present and potential discrete simulations exceeds the reasonable capacity of most present serial computers. The desire, then. is to implement the simulations on a parallel machine. However, certain problems arise in an effort to program the simulation on a parallel machine. In one category of methods, deadlock can arise and some method is required to either detect deadlock and recover from it, or to avoid deadlock through information passing. In the second category of methods, potentially incorrect simulations are allowed to proceed. If the situation is later determined to be incorrect, recovery from the error must be initiated. In either case, computation and information passing are reculired which would not be required in a serial implementation. The net effect is that the parallel simulation may not be much better than a serial simulation.

In an effort to determine alternate approaches, important papers in the area were reriewed. As a part of that review process, each of the papers was summarized. The summary of each paper is presented in this report in the hopes that those doing future work in the area will be able to gain insight that might not otherwise be available, and to aid in deciding which papers would be most beneficial to pursue in more detail. The papers are broken down into categories and then by author.

Conclusions reached after examining the papers and other material, such as direct talks with an author, are presented in the last section. Also presented there are some ideas that surfaced late in the research effort. These promise to be of some benefit in limiting information which must be passed between processes and in better understanding the structure of a distributed simulation. Pursuit of these ideas seems appropriate. 


\section{SUMMARIES OF IMPORTANT PAPERS IN THE FIELD.}

An eflort was made to review the important papers in the area of distributed discrete erent simulation. A few papers were found that were somewhat peripheral to the area and were not examined as thoroughly. Presented below is a smmary of each paper that was read that was found to be pertinent to the subject.

\subsection{Methods Involving Local Clocks and Channel Clocks}

Much of the work in the area of Distributed Discrete Event Simulation (DDES) involving local and channel clocks was done by Chandy, Misra, and associates or was patterned after their work. Papers in this area are considered first. Papers are presented in order by year and sections are identified by the authors of the paper.

\subsubsection{Chandy and Misra, November, 1979}

Chandy and Misla [1] start with a number of definitions:

Definition: A network is a finite collection of processes which communicate with one another exclusively through messages.

Network Representation: The network is represented by a labelled graph in which vertices represent processes and edges represent communication links.

Message Protocol: a message is transmitted along a link only when both processes are waiting to communicate along the link.

Definition: An executable process is one in which some statement in the process can be executed.

Definition: A blocked process is one that is not executable and is waiting to communicate along one or more links.

Definition: a terminated process is one that is neither executable nor blocked.

State transitions take place between executable, blocked, and terminated processes. An executable to blocked transition occurs when no statement in the process can be executed, the process is waiting for communication with another, and the other is not waiting for communication with it.

A Blocked to executable transition occurs when a blocked process is waiting for communication with another, and the other initiates a wait on it.

An executable to terminated transition occurs when a HALT statement is executed and the process ceases to either execute or wait.

A process, once terminated, cannot change state. However, a blocked process camnot terminate (presumably because it needs a stimulus like a message in order to terminate. and that can only occur in the executable mode.) Suspended and mborn processes may be simulated as blocked processes.

Note that messages are buffered by the process, not the link.

Definition: i set of proceses is deadlocked iff.

1. not all processes in the set have teminated, 
2. no process in the set is executable. and

3. that one process is waiting for another process implies the other process is in the set.

Definition: A network of processes is deadlock-free at some stage if no set of processes is deadlocked at that stage. The network is deadlock-free if it is deadlock free at every state of the computation sequence.

Definition: The priority of an edge $e, g(e)$, is a number, not necessarily unique.

Definition: Priorities are proper for an edge $e$ at some stage if for every process $h$ at that stage, $h$ is either

1. executable,

2. terminated, with $g(e)=\infty$ for all edges $e$ incident on $h$, or

3. blocked and waiting on at least the set of edges where the set is the set of finite, minimal-priority value edges incident on $h$.

The highest priority edges are those with minimum priority value. The following theorem is presented:

Theorem: $N$ is deadlock-free at any stage of computation iff. there exists a proper set of priorities $g(e)$ at that state.

For the moment, one might look at the priorities as an order in which messages are sent between processes by priorities placed on edges. If priorities are not unique, then the order in which they are executed is immaterial.

An algorithm for assigning proper priorities at some stage, given a deadlock free situation at that stage is as follows::

Initialization: All executable processes are labelled with 0.

Iteration: At some stage, given edge $e$ incident on $H_{i}$ and $H_{j}, H_{i}$ unlabelled, blocked, and waiting on $e, H_{j}$ is labelled, then give $H_{i}$ the label of $H_{j}$ plus 1 and assign $g(e)$ the value of the label of $H_{i}$.

Termination: Give any unassigned edges that could not be labelled, as above, the priority of infinity.

Concerns raised at this point in the paper are the following: First, what is the significance of the order in which edges are chosen for the iterative step? Does a process become unlabelled when it can no longer execute and must wait? How do we know that $H_{j}$ is ready to supply information to $H_{i}$ ? How is a stage clefined?

The proof states that processes are labelled just once at any stage. One interpretation of the algorithm is that if a process is unlabelled, unblocked, and waiting on two executing processes, then one of the edges must be chosen. This process is labelled with the predecessor process plus one and the edge priority is set to the value of the label of the predecessor process. The other edge would be assigned an infinite priority, since the process became labelled at the first iteration. Thus, with this method of setting priorities, the order in which messages are processed must be unimportant. 
Corollary 1: If at some stage a proper set of priorities exists, and some $g(e)$ is finite, the netrork has at least one executable process.

Priorities change from stage to stage and hence are dynamic, but the authors have not said how.

An example is given of a network of real-time processes which communicate ria messages. Message $m$ at time $t$ depends only upon messages up to time $t-\epsilon$. The example is a job shop, processes are work-stations, and messages are jobs. Jobs are processed in FCFS order. The example contains no loops.

Notation: $A_{i}$ - the time of arrival of $i^{\text {th }}$ job to workstation. $D_{i}$ - the time of departure of $i^{\text {th }}$ job. $S_{i}$ - the processing time of $i^{\text {th }}$ job.

Then $D_{i}=S_{i}+\max \left[A_{i} D_{i-1}\right]$.

This system is simulated by a network of asynchronous processors called logical processors. Messages consist of the 2-tuple $(t, m)$, where $t$ is time and $m$ is message. The dynamic priority of an edge is the time to which it is simulated, i.e.. the time of the last message along the edge, also called the clock value. A process waits on all edges of minimum clock value when it is blocked. In the job shop example, the message $m$ is an integer representing the number of jobs which traverse the edge at time $t$, assuming that all the jobs are of the same type.

The situation can be represented by a number of cases:

Case $1 . n^{t h}$ job departs at or before $t$. The $(n+1)^{\text {st }}$ job arrives at or before $t$. Departures on all output edges up to time

$D_{n+1}=S_{n+1}+\max \left[A_{n+1}, D_{n}\right]$

can be predicted.

Case 2. $n^{\text {th }}$ job departs at or before $t$. The $(n+1)^{\text {st }}$ job arrives after $t$. Then there will be no departures on any output edge before time

$t^{\prime}=S_{n+1}+t$

Null messages are sent along all the other edges when a real message is sent along some edge in order to update the clocks for those edges.

The following procedure is presented:

Procedure: An LP waits on all edges of minimum clock value. If it is waiting on a set of edges, it waits until a message has been completed along all those edges. The message is processed and an output message is developed with an appropriate established time. If a processor is waiting on an output edge, the LP outputs some message. which may be NULL. The authors claim that the waiting condition is sufficient to guarantee absence of deadlock.

That there is no deadlock may not be clear. Consider the following example that appears in another paper. 


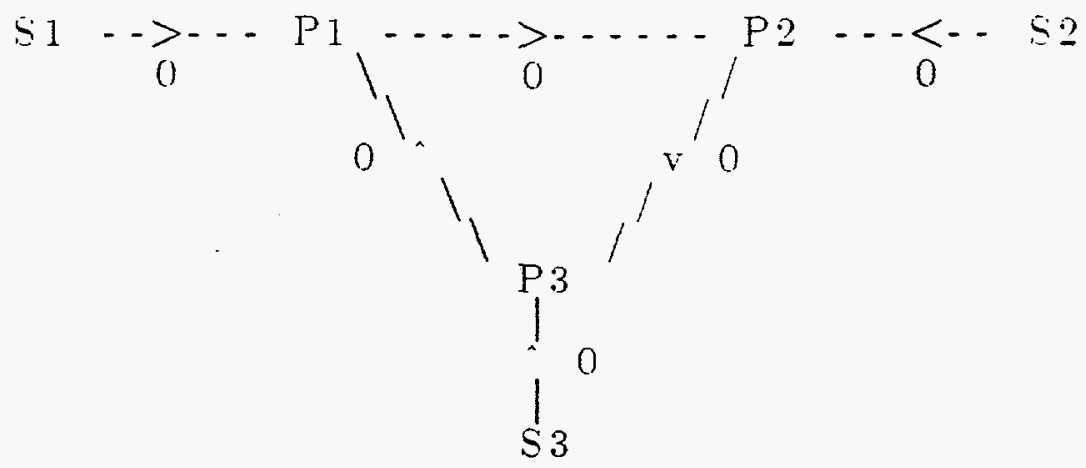

The $S_{i}$ are sources and the $P_{i}$ are processes. All edge clock times are 0 initially. Therefore, each process waits on all of its edges, both input and output. Each receives a message from the source which updates the edge clock. For example, the edge S1->P1 becomes $1, \mathrm{~S} 2->\mathrm{P} 2$ becomes 2 , and $\mathrm{S} 3->\mathrm{P} 3$ becomes 3 . "If it (a process) is waiting on a set of edges $\{e\}$, it continues waiting until message transmission has been completed along all the edges in $\{e\} . "$ We want to process in strict simulation time order. Each process can send a NULL message containing its service time, which is the soonest it can send a real message. Having received a NULL message and updated its edge clock time, it can then determine if it has a real message it can send. If not, it can send another NULL message based on the NLLI. message it has received and can send another NTLL message to again update its cutput edge. As noted in other papers, the existence of at least one proces in the loop with a nonzero service time is sufficient to cause the time to increase to the point were real messages can be sent.

\subsubsection{Chandy and Misra, September, 1979}

Chandy and Misra [2] develop a distributed algorithm for solving a-class of simulation problems. Communication is through messages; there are no shared variables; and there is no central posting or process scheduling.

New messages are dependent only upon messages sent up to $t$ for a message to be sent out at $t$. (One would also assume it depends upon the initial state of the process.)

Physical processes (PP) are simulated by logical processes (LP). PP's that communicate translate into LP's that communicate. There is no global time shared among the LP's. The logical system is asynchronous, and physical time is cncoded in the messiges sent. Also, an LP simulates the corresponding PP and rommingle with other T.P's. Simulation of a PP is independent of the rest of the systent.

Deadlock in distrihted systems is aroided without global coutrol. The alsence of deallock results from the logic of each process.

Nemory is recpired in the logical system to simulate any memory in the fhysical system which storages messages received (if future outputs depend upon jast messages.) The proposed implementation requires a bounded amount of extra storage for each LP.

Represent the system of $N$ physical processes by a directed graph $G$ of $N$ rertices. where there is an arc in the graph iff, the processes communicate in that direction. Message transmission time is assumed to be 0 . Delays can be incorporated at the receiving side or the sending side. Simulation goes from 0 to timc. $Z$. Fach process is assumerl to have some internal delay. Specifically. $T_{k}-T_{i-1}$. 
the time between the sending of two messages, is assumed to be $>\epsilon$, a finite but possibly small number. The stream of messages from the $i^{\text {th }}$ to the $j^{\text {th }}$ process is (haracterized by $\left(T_{1}, M_{1}\right)\left(T_{2}, M_{2}\right) \ldots\left(T_{k}, M_{k}\right)$.

The authors assume that outputs can be predicted up to a time $t^{\prime}$ given the history up 10 time $t$. The value $t^{\prime}-t$ is called the lookahead, $L_{i j}$, for messages along the path i to $\mathrm{j}$.

In the case of loops, the information input may be a function of a previous output of a process. A restriction is made for loops that there exist an arc $\mathrm{i}$ to $\mathrm{j}$ such that $L_{i j}>\epsilon$ for all $t$.

A source process is one which only sends messages and a sink processes is one which only receives messages.

The message protocol is that $L P_{i}$ sends a message to $L P_{j}$ iff. $L P_{i}$ is ready to send the message and $L P_{j}$ is ready to receive it. In addition to normal message, there is a NUiLL message, which does not occur in the physical system. A NULL message sent by $L P_{i}$ to $L P_{j}$ contains only a time value and no $\mathrm{m}$-value as defined next.

Definition: In the tuple $\left(T_{k}, M_{k}\right), T_{k}$ is the $t$-value and $M_{k}$ is the m-value.

Definition: $S_{i j}$ is the sequence of messages sent from $L P_{i}$ to $L P_{j}$ in the simulation.

$T_{\text {ti }}$ is the clock value of line $(k, i)$, which is 0 initially and is the t-value of the last message transmitted to process $\mathrm{i}$ along line $(\mathrm{k}, \mathrm{i}) . T I N_{i}=\min T_{k i}$ over all $k$. Process $i$ can predict all messages to be sent to process $j$ up to $T O U T_{i j}=T I N_{i}+L_{i j}\left(T I N_{i}\right)$ and send them (or a NULL message) with time TOUT $T_{i j}$.

Steps for an LP after initialization until termination are:

1. Select a set of lines for $1 / O$.

2. Compute the tuple to be transmitted to each selected output.

3. Wait to transmit/receive on the selected lines.

If TOU $_{i j}>T_{i j}$, then line $(\mathrm{i}, \mathrm{j})$ is open and $L P_{i}$ can send more messages over it.

$T_{i}=\min \left(T_{i j}, T_{k i}\right)$ over all $\mathrm{j}, \mathrm{k}$ is called the (local) clock value. The set of $\mathrm{I} / \mathrm{O}$ channels is the set of all input and open output lines with clock values equal to the local clock value. For the case of $T O U T_{i j}=T_{i j}$, the line has already been simulated up to that time and $T O U T_{i j}$ must be increased before any further messages are sent.

Determine first if a message is to be sent between $T_{i j}$ and TOUT $i j$ over line $(i, j)$. If so, it is computed and prepared for sending (to be sent when the receiver is ready.) If one is not to be sent, then a NULL message with time $T O U T_{i j}$ is prepared for sending. Following output of the message, the channel time is increased to TOUT $T_{i j}$ if the NULL message is sent, since no message is to be sent before TOLT $T_{i j}$. Otherwise. the channel clock is set to a value $t$ with $T_{i j}<t \leq$ TOUT $T_{i j}$, where $t$ is the time of the message.

Alter the outputs have been determined, it is necessary to wait for processors at the other ends of the selected lines to either transmit messages or indicate willingness to receive messages. This order may not seem right. One would expect to first receive inputs, then compute, and finally to send messages. The reason that it is not this way is that if $T_{i}=T_{i j}$, then a message has already been received on line $(i, j)$ and we are waiting for the next message along that line 
before we can proceed.

Initially. the messages sent for lines $(k, i)$ in process $i$ are the NULL messages (0.NZLL), as are the lines $(i, j)$ in process $i . T_{i}$ is initially set to 0 .

The simulation on a Process $i$ is continued as long as $T_{i}<Z$. When the time for the next message along some line is determined to be $t>Z$, the message (Z.NULL) is sent instead.

The authors provide an algorithm, Algorithm(III-F) in the paper, which they claim is correct, deadlock free, and terminates properly. The algorithm will not be presented here.

The following is an intuitive proof of absence of deadlock by contradiction: suppose deadlock exists. Waiting processors form a loop, since their number is finite. Otllerwise one would encounter a source, and a source will continue to produce messages until termination.) Therefore, the local times must be identical. Howerer. they can't be identical because of the predictability condition.

The purpose of NULL messages is to prevent deadlock which can occur even in an acyclic network without them.

The logical system nerer deadlocks, even if the physical system deadlocks. What happens is that the logical system continues to put out NULL messages with increasing values of time. However, this situation is a correct simulation of the physical system. Time increases without further actual messages being sent. Termination occurs when the time reaches $Z$ for every process.

Performance analysis was obtained for a number of simple systems, all of which are loop free. Increases in speed were obtained.

\subsubsection{Peacock, W'ong, and Manning}

This paper by Peacock, Wong, and Manning [3] is similar to the the Chandy/Misra papers. Of particular interest is the proof as to the conditions for deadlock. It states that "A deadlock exists iff. there is a cycle consisting of empty links which all have the same link time, and nodes which are all blocked because of these links. The following are some observations:

1. A link is empty if there is no message in the message queue for that link. (No customer.)

2. A node is blocked if one of its links is empty and its link time is less than that of all the other links into this node, so that the node cannot determine whether to wait for a message on the empty link or to process a message in one of its link queues.

3. The link time is the time given at initialization or at the last update on that link via a message, which may be a null message and contain only a time update.

4. "Links from a source node ... are not allowed to be empty." One interpretation of this statement is that the links are not allowed to remain empty. That is, the queue may be empty temporarily, but a message is expected over that link, although the time of its occurrence is not known. Therefore, links cannot be blocked because of source nodes.

5. The proof hinges on the fact that the output link time of an empty link from a node is at least as great as the link time of the blocking input link. 
6. A loop must be present.

7. An assumption not stated in the proof but stated earlier in the paper is that a process always sends messages on a link in ascending simulation time order.

Sone observations can be based on the above.

1. If no loops are present, there can be no deadlock.

2 . If we intend to have sources that will cease sending messages, then it is necessary that we send a temination message that allows the receiving node to reconfigure the source out of its list of input links. It also suggests that sources could become bottlenecks, and that it may be desirable to have sources periodically send null messages containing a simulation time if no message is to be forthcoming up to that simulation time.

3. Processes must be designed so that it is impossible for them to send messages out on a link that are not in order of ascending simulation time. Suppose that the process could receive a message in its normal order and send out a message on a link with a simulation time $X$. Now suppose that it can receive a new message and send out a new message on the same channel with a simulation time $Y$ that is less than $X$. Then the second message has caused an output which has bypassed the first. That is, it appears to be simulating processes that can occur in parallel. In that case it ought to be possible to split the process into two processes operating in parallel. Since it is difficult to predict all of the situations that could occur, we must simply leave it up to the designer of the system to assure that messages exit in simulation time order.

4. The theorem proof suggests that if a node has more than one output link, that whenever it outputs a message on one link, that it also output a null message on all the other links that gives the input simulation time of the message that it just processed. The message that it just processed is the one having the minimum simulation time of all the messages in its various link queues. As noted above, the simulation time of any output link must be at least as large as the minimum simulation time of any of the input links. Although this system will not prevent deadlock, it will at least allow some nodes to proceed sooner than otherwise. One would want to make sure that the overhead introduced into the processing did not exceed the benefits.

\subsubsection{Chandy and Misra, 1981}

Chandy and Misra [4] use a deadlock detection scheme to detect and break deadlocks. The concept of a sequence of parallel computations is used. That is, phases of the problem are solved in parallel, where the phases are required to follow one another in a sequential manner. This model is certainly restrictive in the types of problems that can be solved. However, it was read with the hope that it contains some useful jdeas. There is no centralized process that acts as monitor. Termination of the parallel section must be detected in a distributed manner. Termination of a phase is manifested as a deadlock, which is detected in a distributed manner using a modification of a scheme by Dijkstra and Scholten. There is no global clock; instead, each process maintains its own local clock.

Processes in the distributed system communicate via messages. The realizability condition, that the hehavior of a physical process at time $t$ cannot be 
influenced by messages transmitted to it after time $t$, is imposed. Logical processes correspond to the physical processes which simulate the physical process. Nesagre comsist of a pair. (t.m). Where $t$ is the time of the message and $m$ is the monchuge itself.

Recuirements:

1. Messages along a link are sent in simulation time order.

2. There must be a one-to-one correspondence between the messages sent in the physical system and their actual time and the messages sent in the logical system and the simulation times, with the messages containing the same information and the times being exactly the same for both.

3. The order in which messages are sent between physical processes and their corresponding logical processes must be the same.

The meaning of time $T_{k}$ in the $k^{\text {th }}$ message from $L P_{i}$ to $L P_{j}$ is that $L P_{i}$ has simulated all messages from $L P_{i}$ to $L P_{j}$ up to time $T_{k}$.

Bounded buffers are used for communication. Specifically, buffers of size 0 are used in the paper. $L P_{i}$ transmits to $L P_{j}$ only if $L P_{i}$ is waiting to send and $L P_{j}$ is waiting to receive.

The clock value $T_{i}$ of $L P_{i}$ at any point in the simulation is the maximum time that satisfies the requirement that all subsequent messages $(t, m)$ sent or received by $L P_{i}$ must have $t>T_{i}$.

\section{Consequences:}

1. $L P_{i}$ must have received a message along each input line with a tcomponent greater than or equal to $T_{i}$.

2. $L P_{i}$ must have deduced that it will not send any message $(t, m)$ on any output line where $t \leq T_{i}$.

The clock value of a line $(i, j)$ for $L P_{i}$ is the lower bound on the $t$-component of the next message transmitted by $L P_{i}$ to $L P_{j}$ along that line. The clock value of a line $(i, j)$ for $L P_{j}$ is the t-component of the last message transmitted by $L P_{i}$ to $L P_{j}$ along that line. The channel times computed by $L P_{i}$ and $L P_{j}$ may not be the same.

The operation of an LP is to alternate between computing and waiting, and uses the following rules when waiting:

1. $L P_{i}$ waits to receive on all input lines whose clock values equal the local time.

2. LP; waits on all output lines for which a message is to be sent.

The authors show a deadlock situation due to a merge operation. The deadlock is looked at by the authors as being due to a loop (here created by a pair of feedfomard branches) in which waiting and not waiting alternate along the paths of the loop. Deadlock is aroided by having processors send NULL messages, $(t$. V $L L L)$. signifying that $P P_{i}$ does not send $P P_{j}$ any message in the time interral betwcen the last message along $(i, j)$ and $t$. Specifically, $P_{i}$ would send such a message along all other output lines when it sends a real message along one of them. Note: deadlock would not have occurred in the example given in the paper 
were it not for the protocol that required the sender to store a message until the receiver was ready to receive it. If buffers are allowed at the receiving end and if processcs could send a message when ready, no deadlock would occur. A feedback type loop is necessary for deadlock to occu in the infinite buffer case.

Many cases are shown in the example where null messages need not be sent. These are cases in which no merge operation exists downstream. They may be too infrequent to worry about.

The authors recognize that there may exist a propensity of NULL messages, and that feedback paths further complicate the situation. They note that there is no mechanism for annihilating NULL jobs. Perhaps one exists, however.

The authors propose a distributed scheme for detecting and recovering from deadlock. The algorithm is as follows:

1. Parallel phase: Run and simulate until deadlock.

2. Phase interface: Initiate a computation whereby the various LP's can advance their clock values.

The above actions are synchronjzed by using a controller. The controller detects deadlock based on the termination detection algorithm of Dijkstra and Scholten. The controller directs the deadlocked LP's to perform the phase interface computation. When completed, the LP notifies the controller and continues parallel computation. The controller detects termination of one phase of the parallel computation and initiates the next one.

The examination of this paper was terminated at this point because it is similar to other papers by these authors. The approach appears to be too limited in application.

Comment: The waiting concept may be helpful in understanding what is happening. However, it seem more time efficient to send a message when it is ready to be sent. Many computer systems have transparent message transmission; that is, the operating system of one processor will communicate with the operating system of the other processor and arrange for the message to be transmitted into a buffer, which may take place via a DMA operation. The message is then available for the receiving process to examine when it is ready to do so.

\subsubsection{Smoliar}

Smolini [5] proposes a technique in which software and supporting operating system constitute two levels of networks. Communication is by message exchange, both within a single level and between levels.

The authror suggests that one way to confront the interactions between software and its operating enviromment is through the use of data-driven models. In data criven modeling, any system is regarded as a network of communicating processes, or configuration. Software and operating environment may be regarded as two levels of configurations.

A process is represented by a graph, and a configuration is represented by a collection of processes. It seems more appropriate to represent a configuration as an interconnection of processes. A process consists or either a function or a connection to another configuration. a synthesizer, and one or more channels. A channel can be either internal, i.e., an interconnection to a process within a configuration, or external, i.e.. a connection to a configuration embedded in another process. Note: a configuration must always have exactly one external 


\section{OLTPUT channel.}

Processes are modeled by continuous functions over sequences of messages. Specifically. the process maps the history of input messages on the input channels to the history of output messages on its output channels. These channels appear as several nodes in a configuration, i.e., as an output node of a process and as one or more inputs nodes to other processes.

A function is a simple transducer that takes an input message and converts it to an output message, independent of the past input history. It appears that it must at least be a function of the time associated with previous messages. A synthesizer develops an output message based on past history and present input messitge.

Every channel is an unhounded queue. Function outputs go to the output chammel at the tail end of the queue. (lt is not clear, but it appears that where the channel connects to several other processes, each process receives the message.) Synthesizers receive messages for the heads of their respective input channels on command and send their outputs to their corresponding functions. From the description of how a channel may provide input to multiple processes, that is, to replicate the queue once for every process, it appears that the message is indeed sent to each of the processes. However, a process may have more than one output, so the above is not a limitation.

Physical resources and facilities provided by the operating system may be viewed as networks of communicating processes. Simply view the resources as state machines that accept, as inputs, messages from other components and use these messages to update their state and produce outputs which are passed as messages to other processes. Even a memory can be considered a process.

A compiler takes each process and translates it into a collection of disjoint modules that run under a message-oriented multiprocessing operating system in the actual physical computing environment. This compilation may be defined in terms of physical nodules or logical modules, where the former implements the configuration anc he later serves as a simulation of the configuration.

A physical module is nothing more than another state machine, i.e., it receives its inputs in the form of messages from other physical modules. Only the modules which implement synthesizers need receive inputs from multiple modules. State and input are then used to determine output which is sent to one or more destination physical modules.

Logical modules differ from physical modules in the way that they advance logical (simulated) time. As with Chandy and Misra, time is distributed among the logical modules; hence the system has no global clock. Each module has its own logical clock. Each message has a timestamp, which is the value of its logical clock at the time the message is sent. These timestamps are used to synchronize operations.

The recognized problem occurs with the synthesizer, because it is allowed Iu have more than one imput channel. The synthesizer performs a "nondeterministic" merge among it multiple inputs. These inputs to the logical module must be processed in timestamp order.

Each function, synthesizer, input channel, and output channel are translated into a unique program module and these modules exchange messages according to the connections defined by the configuration. A function module cycles through the states "receive input from synthesizer," "perform function," and "send output to output channel." The "perform function" operation of the logical function module is assumed to include the handling of the timestamps. How the function handles the timestamps is not specified. at least up to this point in the paper. A 
synthesizer maintains its own set of buffers, one for each input channel, and requests for data from an imput channel are directed to the buffer. If a buffer for an input channel is empty and a request is made to that channel, then the synthesizer waits until a message arrives, whereupon it performs the synthesis function. The manner in which a channel is chosen is not specified.

The injut channel function simply receives input from the source and sends it to the srnthesizer. The output channel of a function receives input from the function and sends it to all the destinations.

If a synthesis module is connected to an embedded configuration, it will send its outputs to the appropriate external input channel module. The corresponding output channel module will receive its inputs from the appropriate external output channel module.

The software simulation interacts with the operating environment by sending and recejving a message. One of the primary functions of this message exchange with the operating environment is the advancing of the logical clock in such as way as to model the time consumed by the use of its corresponding environment facility. Messages sent to the operating environment will be tagged with the source to assure that the return message is sent to the correct module.

The author of this report feels that some key questions are skirted and not answered, the most specific being how the synthesizer orders the messages by time, and how deadlock, if possible in the system, is detected and broken.

\subsubsection{Yamamoto and Hasegawa}

Yamamoto and Hasegawa [6] consider an algorithm for advancing time in a fully distributed discrete event simulation on a multi-processor system called KDSS-I. It is an object-oriented system, and uses elements called QSVs, which correspond to logical resources. A simulation clock is distributed to every QSV. Deadlock is detected via a loop algorithm and a method is presented for handling complex loops.

In their view, a model consists of active elements (resources) and passive elements (transactions). A basic active element is a queue followed by a server. This element, a QSV, controls simulation time and events. Information is passed between QSVs via messages. A message containing information is termed an actual packet. A special class of message is the time packet, which contains only time.

A QSV has two clocks, a receive-side clock and an send-side clock. Two special active elements are a generator, which acts as a source and generates message pacliet but has no inputs, and a terminator, which acts as a sink and "destroys" packets but has no outputs.

Two basie assumptions are:

1. All input lines must have packets.

2. The packet with the oldest timestamp is chosen to be processed next.

These are basic assumptions with the Chandy/Misra method. Once a packet is chosen for processing, the receire-side clock is advanced to the timestamp of the packet being processed, and the send-side clock is advanced by the waiting plus service time. Where waiting time is determined based on the arrival time of the message and the departure time of the last packet. The timestamp of the packet being sent is that calculated for the send-side clock. A time packet containing 
this same timestamp is immediately sent to all the other QSVs to which it outputs packets. In this way, these successor QSV know that they cannot receive a message with timestamp less than this value along that channel. Misra would refer to these time packets as null messages. Although that authors do not so state, one might assume that time packets are accepted for processing in the same way that actual packets are chosen for processing. A time packet will change the receive-side clock, unless the two times are the same. The authors are not clear on what process is performed by the QSV when a time packet is selected. What makes sense is for the process to send out a time packet of its own to all its immediate successors if the received time exceeds the send-side clock, since the successors can then update their clocks. The send-side clock can be updated to the new receive-side clock time.

The null messages described above are not sufficient to avoid a deadlock situation when a loop is present in the process graph. The authors next consider a simple loop. The possibility of deadlock is detected by the fact that "a QSV accepts a time packet but cannot advance its clock." The authors claim that "If there is no time packet which can advance clocks and bring an event into a loop, only time packets having the oldest time exist." The meaning of the authors is not clear. ('The detection method appears flawed, in that a process may receive a mosage. he able to process it, have messages waiting, but yet not be able to process any messages because some channel does not have a message on it.) Detection of the deadlock consists of a QSV sending out a detective message with time information and ID. This message is sent around the loop, and if the time remains unchanged by another process in the loop, deadlock is present. Such a step attempts to determine deadlock as defined by Misra. In that case, the detective message contains the receive-side clock for the initiating process. The authors, in fact, desire to determine the process and associated time for the next event within the loop. Therefore, they take a different approach as discussed next.

Define Next Event Time (NET) for a QSV as the oldest time of any packet on any channel on the QSV such that the channel originates outside the loop. Define NET for the loop as the oldest NET of any of the QSVs in the loop. The QSV that originates the detective message sends its local NET. When a process selects a detective packet, it compares the local NET with the NET in the detective message. (The authors do not state the criterion for selecting detective messages. Presumably, if the process has messages that it can process, it will ignore a detective message, since deadlock is not present.) If its own NET is older, then it replaces the NET in the message with its own NET. When the message reaches the originating QSV and the time has been unchanged, then the originating process may process the message with that time-stamp because it is the oldest message of any associated with the loop.

Some questions arise. Can we be assured that every channel, with the possible exception of the channels associated with the loop, will have a message? The authors do not present an argument to this effect. Will the condition exist, as stated above, for initiating the detective message when, in fact, deadlock exists? The authors do not present a proof. Can a detective message be initiated without deallock existing, and if so, what should be done? Here, the authors are silent.

Assume that all is well so far. The authors discuss the case of more complicated loops. A QSV at which two or more loops merge is called a joint QSV. A tree-like loop, is a set of simple loops connected with each other like a tree. I take this statement to mean a root loop can be identified, leaves can be identified, and that for erery pair of loops, the pair intersect at only one process. The authors do not adequately define cyclic loops. Perhaps one can take cyclic loops to be 
those that do not fit the tree-like loop definition.

Tree-like loops can be "reduced," starting with a leaf loop. A leaf loop is one that intersects only one other loop. The authors state that an NET can be found for this loop by sending a detective message around this loop. The same questions noted above apply here. Ther further claim that the loop can then be considered to be equivalent to one QST: This process can then be repeated until only one looj remains. Presumably, this loop can be treated as was the simple loop above. Howerer. much detail remains unspecified.

Complex loops cannot be reduced as above. The authors propose a method of "untying" the loops. Suppose two definable loops meet at a process. Then inslead of treating these as two separate loops, one can treat them as a single loop in the following way: Start al any point on either loop. Traverse the loop until the intersecting process is reached. Then traverse the other loop back to the intersecting process. Then traverse the remaining part of the loop. This process essentially eliminates intersection points. The authors seem not to consider the case in which two loops intersect at more than one process. (Think of two overlapping loops.)

What is proposed by the authors is very similar to previous proposals for detecting and breaking deadlock. Questions remain as to whether the method works in all cases. Furthermore. for a process graph that is very complicated in terms of loops, the detection and breaking of deadlock appears to be a complicated process. Finally, it appears that the detecting and breaking of deadlock is not transparent to the user.

\subsubsection{Chandy, Misra, and Haas}

The paper by Chandy, Misra, and Haas [7] specifically presents distributed deadlock models for resource and communication deadlocks. An assumption made by the authors is that message delays are arbitrary but finite and there is no single central agent. Messages sent by one process to another are received in the order in which they were sent. Two model are presented, the resource model and the communication model.

The resource model assumes that a process requesting resources must wait until it acquires all the requested resources before it can proceed with its computation. In the communication model, resource requests are specified as a logical expression involving the recuested resources and the operations AND and OR. When it receives one of an alternate set of resources, it must cancel the other requests. The authors claim that all genuine deadlocks are detected and no false deallocks are reported. In the communication model:

1. A process may wait selectively for messages from some other processes.

2. Any process may send a message to another process without having received a message.

Thus deadlock detection involves the detection of a subset of processes waiting for each other.

A set of processes communicating with each other via messages is a network. A process never waits to send a message. If synchronization is desired, the sending process waits for an acknowledgment.

The description of a Resource Model is aimed toward distributed data bases and was not st udied. 
Communication Model: The model has no explicit controller (or resources); controllers must be implemented by processes and requests for resource allocation, cancellation. and release must be implemented in messages.

An idle process starts executing upon receiving a message from any process in its dependent set. A process is terminated if it is idle and its associated dependent set js empty. Conceptually. a set of processes is deadlocked if all processes in the set are permanently idle. i.e.. they never receive a message from any process in the dejendent set. Deadlock cannot be detected if one of the processes in the set is executing.

Formally. a nonempty set of processes. $\mathrm{S}$, is deadlocked iff.

1. All processes in 5 are idle.

2. The dependent set of erery process in $\mathrm{S}$ is a subset of $\mathrm{S}$.

3. There are no messages in transit between processes in $\mathbf{S}$.

A process is deadlocked if it belongs to some deadlocked set.

It is important to note that the authors specify the difference between the resource model and the communication model as the difference between waiting for all resources and waiting for any one message. If the problem is viewed in terms of a process graph, deadlock arises in the resource model when there is a cycle of (idle) dependent processes; whereas, in the communication model there must be a knot of idle processes. (A knot in a graph is a vertex $\mathrm{i}$ of a directed graph such that all vertices that can be reached from $i$ can also reach $i$.)

Deadlock Detection in the Communication Model: An idle process initiates a query computation when it enters the idle state. Such messages can be processed by an othemrise idle processor. The initiating process is called the initiator. A query and a reply to a query contain the 4-tuple ( $\mathrm{i}, \mathrm{m}, \mathrm{j}, \mathrm{k})$, where $m$ identifies the $m^{\text {th }}$ query initiated by the process $P_{i}$ with the message being sent from process $P_{j}$ to process $P_{k} . P_{i}, m, P_{j}$, and $P_{k}$ are initiator, sequence number, sender, and receiver, respectively.

A query computation has the following property: $P_{i}$ is deadlocked when it initiates its $m^{\text {th }}$ query computation iff. it receives reply(i,m,j,i) corresponding to every query $(j, m, i, j)$ that it sent.

The algorithm requires four local arrays: latest(i), engager(i), num(i), and wait(i), where

latest(i) is the largest sequence number $m$ in any query $(\mathrm{i}, \mathrm{m}, \mathrm{j}, \mathrm{k})$ sent or received by $P_{k}$. (Initialize to 0 .)

engager(i) for $i \neq k$ is the identity of the process which caused latest(i) to be set to the current value by sending $P_{k}$ the message query $(\mathrm{i}, \mathrm{m}, \mathrm{j}, \mathrm{k})$, in this case $\mathrm{j}$.

num(i) is the total number of messages of the form query(i, m, $k, j)$ sent by $P_{i}$. minus the total number of messages of the form reply $(\mathrm{i}, \mathrm{m}, \mathrm{j}, \mathrm{k})$ received by $P_{l}$. where $m=$ latest(i) and $\mathrm{j}$ is arbitrary.

wait(i) is true iff. $P_{k}$ has been idle continuously since latest(i) was last uprated. (Initialize to false for all i.)

A]gorithm:

An idle process initiates a query computation by sending to processes in its dependent set. Receiring processes, if idle, propagate the query to their dependent set (if it has not done so already). and so on. 
Let latest(i). engager(i). num(i). and wait(i) be local variables of process $P_{k}$. Also. let process $P_{k}$ receive a message with initiator i. The $P_{k}$ takes actions as follows: If $m<$ latest(i). then $P_{k}$ discards the message, since latest(i) indicates $P_{i}$ initiated another query after sequence number $m$. Therefore, it could not have been deadlocked when it sent the message with sequence number $m$.

If wait(i) is FALSE when $P_{k}$ receives the message AND $\mathrm{m}=$ latest(i), then by the definition of wait(i), $P_{k}$, has been busy at some time after the message, with sequence number $m$, was received. Because $P_{k}$ has been busy at some time in the interval, $P_{i}$ could not be considered deadlocked because a member of its dependent set was not continuously idle. Thus, $P_{k}$ would discard the message.

Idle process $P_{k}$ discards all messages except those for which $m>$ latest(i) or those received when wait(i) is TRUE and $m=$ latest(i).

Case 1. $m>$ latest(i) $-P_{k}$ sets latest $(\mathrm{i})=\mathrm{m}$, engager(i) $=\mathrm{j}$ (where $\mathrm{j}$ is the sender), and wait(i) $=\mathrm{T}$

Case 2. $\mathrm{m}=$ latest $(\mathrm{i}) \mathrm{AND}$ wait(i) $=$ TRUE $\mathrm{AND}$ message is a reply $-P_{k}$ decrements num(i) by 1 .

$P_{k}$ propagates queries in which $m>$ the number of latest(i) to all dependent set, sets num(i) = \# processes in dependent set, and updates other local variables.

$P_{k}$ sends replies to a query as follows:

Case 1. immediately when wait(i) = TRUE AND $m=$ latest(i) with reply $(i, m, k, j)$ - Since $m=$ latest $(i)$, this is at least the second query received designating $P_{i}$ and $m$. It appears that every process must receive at least two queries regarding processor $P_{i}$ and $m$.

Case 2. never if wait(i) is FALSE and $m=$ latest(i) OR $m<$ latest(i).

Case 3. to engager(i) only if wait(i) $=$ TRUE AND num(i) $=0$.

The statement is made that "if the initiator receives replies to all the queries it sends to its dependent set, then the initiator is deadlocked." A busy process stores up any messages it receives while in the busy state, but discards them when it becomes idle, since it was not deadlocked when the messages were sent. It appears that there is not a problem if the process does not send a data message before it becomes idle, because when it becomes idle, it initiates a query. However, does the sequence in which a process receives query and reply messages have an effect on the outcome? For example, suppose $P_{k}$ is in the dependent set of $P_{i}$ but receives a message from $P_{j}$ with initiator $P_{i}$ and number $m$ before it receives a message directly from $P_{i}$.

How are queries stopped from continuing to cycle ad infinitum? An initiator can recognize itself as being the initiator of the original query and not pass it on to its dependent set. An other process passes on the query the first time it reccives a query with a given initiator and sequence number and records receipt of that query. If it receires additional queries with that same initiator and sequence number. it simply responds to them.

One of the assumptions with the communication model is that any message sent by one process to another is received correctly after an arbitrary but finite delay. and messages ober the first-in-first-out rule. Can the message delay cause a problem. in that a message could be in transit, but not yet received? In that case, the process might think it is idle, but it has a message on the way to process. From the procedure described above, the order in which queries are received is unimportant, since the process passes on queries only from its engager, as indicated by engager(i). 
Theorem 3, that states that at least the last processor to become idle will dcclare itself deadlocked. appears correct. If $\mathrm{it}$ is the last one to become idle, there can be no more real messages in the set $S$. Thus, an alarm will be sounded. Can there be any false alarms due to message delay? For this to happen, a process would have to claim it is idle. but then receive a real message. I think the only way that could happen is if a message from a source arrives out of order, and that is not allowed by assumption. Therefore, any system that sends messages from one point to another must assure that the messages from that source are received in order. If the operating system keeps the correct order, then the only way messages could be received out of order is if two or more paths exist between the processes. Problems could arise in a hypercube, where messages could be sent by different routes, or in networks of processors, where more than one route exists and store and forward is used.

The proofs to Lemma 1 and Theorems 2 and 3 also appear to be correct. However, the proof to Theorem 1 refers to a proof in another paper, and that proof has not been verified.

The authors do not indicate a scheme for breaking deadlock once detected, except for the statement that "any process detecting deadlock can inform others that it is deacllocked."

The authors state a number of reasons for feeling that their algorithm is a good one. One is that the overhead of deadlock detection computations and the message traffic associated with deadlock detection is generated primarily when processes are idle. However, that is a misleading statement. In many parallel machines, e.g., a hypercube, the computer that is sent a message must spend time receiving the message, even if it does not process the message. If the sending and receiving processes are not logically adjacent, the message must be routed through one or more intermediate nodes. In each case, time may be taken away from a busy process to handle messages for which it specifically has no use. Furthermore, a large number of processes could go idle almost simultaneously. If that is the case, then the number of queries and replies taking place as a result can be significantly more than what can be expected from just one process going idle. A result of this situation is that those few processes that are busy would be burdened down even more so by accepting and relaying messages from processes that have gone idle.

The authors suggest the possible use of a timeout parameter, T. A query is only sent if a time of T has elapsed after the process has gone idle.

We have here a rather elaborate method of detecting deadlock. According to Chandy and Misla, deadlock does not exist unless there is a loop in which no process introduces delay. Thus, we can eliminate deadlock by introducing delay into one process in every loop, which in terms of real systems is reasonable. However, null messages are required, and if the delay is small, the number of null messages can become quite large. In addition to the elaborate scheme of deadlock detection, some scheme must be introduced to break that deadlock, and the authors do not state a method of breaking the deadlock.

\subsubsection{Yoshida and Tokoro}

The method presented in the paper paper by Yoshida and Tokoro [8] purports to manage time for a discrete system simulation. Deadlock prevention and detection methods are given. There is no global clock.

System components to be simulated are referred to as objects, which have attributes, activities, and simulation clock. The first kind of interaction between 
objects is by state transition, which is a system-wide event occurrence. The two possible states are active and passive. An object in the passive state is activated by an activate command, which includes a time $t$. An object is placed in the passive state with a passivate command. Both state transitions are executed by a communication command.

The second type of interaction is by data exchange, in which global or remote rariables are "accessed." It is performed via the execution of an access command. Access commands are of two types. read and update. The simulation system is assumel not to have common or shared memory. Therefore, communication is ria nessagus. Any object that reads oj updates a variable is assumed to have a copl of that rariable.

One aspect that seems different than with other methods is that both server and served are considered as being objects. In our implementation of the barbershop problem, for example, the barber is a server and is viewed as a process. The customer is viewed as an object and moves through the system in the form of a message. In the system described, both barber and customer are viewed as objects and can be activated and deactivated. For example, the arrival of a customer at the barber queue would activate the barber, if inactive, and deactivate the customer. When the barber has serviced the customer, the customer is reactivated, and the barber is deactivated, unless there are additional customers waiting.

In order to obtain a correct simulation, each object must simulate its events in the proper order, and the simulation time of each object must be monotonically nondecreasing.

An active interaction point involves either an activate or an update command. where the activation command is a message with a timestamp and the update command involves sending messages to each object that commonly reads that variable. A passive interaction involves either a passivate command or a read command, where the passivate command performs a synchronization operation with those objects which may activate this object and the execution of a read command causes the object to perform a synchronization with those objects which may update this variable.

Objects possess activate message lists, which correspond to passivate commands, and update message lists, which correspond to variables. Received messages, with sender IDs, are linked to the appropriate list. The object contains a list of objects that can activate the object after a specific passivate command and a list of objects that can update a variable after a specific passivate command. The authors state that the compiler can determine what objects may send the messages. These lists sound much lilie the Misra channels, except that here the lists appear to be dynamic and the lists for activate and update need not be the same.

An object which receives an activate command message links it in timestamp order to the appropriate activation message list. It is not clear at this point how the appropriate list is determined. An object which receives a passivate command determines the timestamp of the earliest message from those in the appropriate actiration message list. It then determines which objects can activate it from the corresponding activation object list. If activation messages are not pending from some of these sources, a time-query message is sent to each such object. The object sends an activate reply when its time is, or becomes, greater than the recuesting object's time unless it sends an actual activation message before reaching the regursing object's time. 
When a variable is updated. the object sends an update message containing the name of the variable, the value, and the object's current time. The receiving olject links the message with the update object message list in timestamp order.

When a variable read command is issued, the most recent update of the variable must be determined. The procedure is the following: The objects that can upclate the message are determined. The update message list is then checked to determine those having a time earlier than the object's time and whose last update time is unknown, because there is no update message in the list. These are sent a time-inquiry message. Here the authors are not clear, either in the written text or the accompanying program, how the object responds to the time-inquiry message. In order that the rest of the algorithm function correctly, the receiving object must wait until its time is greater than the timestamp of the time-inquiry message. When it has received replies to all such inquiry messages, it is free to choose the message on the list having the most recent time-stamp less than its orn time. It can then discard older messages, update its own copy of the variable. and read the variable. This process seems to be a very elaborate one in order to be able to determine the value of a variable. Note that messages from an object to another object must arrive and be processed in the order in which they are sent from the object. The necessity to wait for inquiry commands means that deadlock can occur. The authors state the following necessary and sufficient conditions for deadlock to occur:

1. Existence of a cycle in the clirected object graph. The graph is assumed to show message paths as arcs and objects as nodes.

2. Each object in the graph executes the following secuence of commands:

a. a passivate command which will be activated by a predecessor object in the cycle, or a read command for a variable which will be updated by a predecessor object in the cycle.

b. an activate command which activates a successor object in the cycle, or the advance of time which releases the successor object in the cycle which is waiting in a read command.

3. In the cycle, the following relation holds among objects which update or read the same variable

update time $\leq$ read time

No proof is given of the above assertion.

The authors suggest a way in which deadlock can be avoided in certain cases; howerer, they acknowledge that deadlock may not always be prevented. When deadlock cannot be aroided, they suggest the use of a deadlock detection token. A deadlock detection token is given to each cycle that can satisfy conditions 1 and 2. When condition 3 holds in the object that has the token, it passes the token back to the predecessor object with its current timestamp. The predecessor object passc lie loken back to its predecessor if this object is in the wait state at the ime equal to the value of the timestamp in the token. Otherwise it keeps the token. If erer it enters a wail state. it passes the token back to its predecessor witl a timestamp equal to its current time. Passage of the token back around the loop without it being reserved constitutes deadlock. The authors don't say what to do if deadlock is detected. The statement of what constitutes deadlock detection does not seem quite correct. A reasonable interpretation is that if the token returns to an object without the time being changed, then deadlock is detected. 
A serious failure of the paper is the lack of a method to break deadlock once it is detected. Another failure is the lack of any proof that the conditions given for deadlock are necessary and sufficient. The paper does not indicate some underlying assumptions, e.g., that messages from one object to another must arrive in the order in which they are sent. The number of messages can become quile significant. In some systems where message communication overhead is significant, the sending of messages with a single variable can be quite expensive in time. Where complex loops exist, the determination of how to route tokens may prove difficult. The updating of variables appears to be a significantly time consuming operation. The "complier" will need to be rather complex in order to determine objects which can send messages to other objects and to detect the crcles that can exhibit deadlock.

\subsubsection{Reed.}

The paper by Reed [9] presents three performance studies based on a prototype implementation of one parallel simulation algorithm. The algorithm is the Chandy/Alista DDES algorithm.

One of the goals of distributed simulation is to eliminate the event list, used in the usual approach to serial simulation. Server/queue pair are mapped into individual processors in the distributed system. Each processor operates its own simulation clock, and communication is through messages.

The author notes certain problems with distributed simulation.

1. Inter-processor networks are required, and the author believes these to be currently in the design state and not currently available. (The author of this report disagrees.)

2. Assignment of server/processor pair to processors can be difficult.

3. Only a subset of all DES models are amenable to distributed simulation.

4. Deadlocks occur in most simulation models.

Two alternatives exists in the assignment problem. One can fix the topology and map queues onto processors, but optimizing the assignment is an NPcomplete problem. Alternately, one can reconfigure the system topology to match that of the network of queues. The author feels that doing so requires rewiring the network for each simulation model, which may not be true. Such could possibly be done in software.

In the implementation of the Chandy/Misra Algorithm, five node types are defined: service, fork, merge, source, and sink. Service nodes correspond to queueing network servers, fork and merge nodes provide routing, and source and sink nodes create and destroy network jobs. The above constitutes part of the RESQ scheme.

Deadlock is handled via one of two schemes: the sending of null messages, as suggested by Chandy and Misra, or by the detection of and recovery from deadlocks. Chandy and Misra have a distributed scheme for the latter.

The central server model as proposed by Buzen is used. This model is shown in Figure la of the paper.

The remainder of the paper is addressed to performance. The models are rather simple models. The distributed simulation was not performed on a real system, but was itself simulated. Most of the routines show less than a two-fold speedup for a 50 node case. The author attributes the poor performance to the 
lact that the code was written in FORTRAN and FORTRAN does not have a synchronization mechanism for multiple access to the same data structure, so that secuential code was generated. The author is not convincing.

'The paper does not give any new insight or methods for handling the major wobloms with the Chand\%/Alism method of DDES.

\subsubsection{Kumal}

This payer by Kumar [10] only considers the case of feedforward systems. That is. cycles are not allowed. The omission of cycles greatly limits the systems that can be simulated by Kumar's methods. However, it was read because it might contain some new ideas. Communication is via messages, and there is no shared memory or global clock. The messages exchange information about events and their time of occurrence.

The new distributed simulation scheme is called TBASIC. Although it simulates feedforward schemes only, it involves almost no overhead messages.

A physical system consists of a finite number of physical processes (PPs) that interact with each other at cliscrete moments of time by exchanging messages along links that are unidirectional, unbounded, and have 0 delay. (The term unbounded is not defined, nor was it inferable from the remainder of the article. One reasonable interpretation is that a link is capable of storing an unbounded number of messages.)

The message history of a line $(\mathrm{i}, \mathrm{j})$ from process $\mathrm{i}$ to process $\mathrm{j}$ up to time $t$ is the r-tuple $\left.<\left(t_{1}, m_{1}\right),\left(t_{2}, m_{2}\right), \ldots,\left(t_{r}, m_{r}\right)\right\rangle$, where $\mathrm{m}$ 's are messages and times $<t$ are times at which messages were sent. If the message history up to time $t$ is known for all links to a PP, then the output message history for the PP can be computed up to at least time $t$.

The PPs considered by Kumar are: delay, merge, fork, source, and sink. A delay is a FCFS queue with attached server, where the service times may be deterministic or probabilistic. The merge PP routes input messages on a number of lines to a single output line. Whenever it receives a message on one of its input lines, it sends it via its output line after zero delay. If two or more messages are received at the same time, they are put together in a single message and sent out via the output line. The fork routes its single input line messages to one of its output lines, with the output line being chosen probabilistically. A source generates messages and a sink absorbs them. Limiting the simulation process to the above five elements further restricts the kind of systems that can be simulated. Because of the way the merge operation works, the physical system must operate in real time. The restriction of the fork operation to probabilistic decisions seen unnecessarily restrictive.

The physical system is simulated by a distributed simulator called a logical system, which is a network of processes called logical processes (LPs) that are topologically isomorphic to the physical systems they simulate, with each PP being replaced by one LP.

The sequence of pairs $\left(t_{k}, m_{k}\right)$ sent along any given line $\mathbf{i}, \mathbf{j}$ must be sent in strictly chronological order of the $t$ values. The process must assure that if the order holds on its input lines, it holds on its output lines. This requirement is common with Misra-type methods. However, the author does not state how chronological order is to be maintained for the LP corresponding to the merge PP, simply that it must be done. One can only assume that the author intends to use a molhoul such as Misrats mothol to assure the order. 
Temination for a process occurs when each input line has a t-value componding to the termination time $Z$. where some of the messages may be termination messages with time $Z$ and a null message. No message is sent if the time for the message is $>Z$. If the message calculated for an output line is $>Z$ or the process lerminates and the last message sent on that line has a time $<Z$, then the process must send a termination message to its successor.

A syrtem is safe if at any point in the simulation, the pair sent or received on a line correctly simulates the sequence of messages sent on the corresponding physical sytem up to a certain time, i.e. that they are sent in chronological order of their t-ralues. Also. there is a $1-1$ correspondence between messages sent in the simulation and in the physical system. except for the termination message, for each line up to time $t$. A system exhibits the liveness trait if each line in the system receives a message having time $Z$ in a finite time from the start of the computation. The proof regarding safety is missing, and would depend upon how a merge operation is simulated in the corresponding LP, which (as stated above) is not given.

The authors prove that the system they propose is deadlock free. The proof is based on the fact that the system is acyclic. This conclusion is consistent with facts concerning deadlock established by Misra.

There seems to be little new in the paper, and important details are missing. The model is unduly restrictive, and few real systems would fit within the model. some of thesc have been mentioned above, e.g., the restriction to acyclic systems. Another is that the fork operation does not allow a situation such as our barhershop example, in which customers are sent on a first available basis to the barbers.

\subsubsection{Misra}

This paper by Misra [11] brings together a number of the previous papers published by Misra and his associates. It specifically discusses deadlock avoidance and deadlock detection. It is billed as a survey that presents an "entirely new approach to the problem of system simulation."

Messages are again assumed to be the mode of interaction between autonomous physical processes. If the physical processes do not actually interact via messages, they can often be modelled in those terms. The method is directed to discrete event simulation.

The method has no shared variables such as a clock or event list. Machines simulate physical processes and messages between them simulate messages in the physical sysiem. with time encoded into the messages.

Deadlock is recognized as a possible problem with systems that use the message passing scheme, and different techniques for distributed systems have been proposed for dealing with deadlock and termination detection.

Physical processes (pp's) represent components of the real system to be simulated. Each is described by a set of events, and each event has an associated time. Events generally depend upon the outcome of other events and are dependent in that respect. An event cannot be processed until all events upon which it depends have been processed. pp's operate autonomously and interact by sending and receiving messages. The author assumes that message delay is zero. Delays could be modelled at either the sending or receiving end.

The author introduces two concepts inherent in physical systems: realizability and predictability. The concept of realizability is that in a real system, a message sent by a pp at time $t$ is a function of its initial state, $t$, and the messages it has 
receired up to and including $t$. That is. a pp cannot guess any message it will receive in the future.

As an aside, recognize that it is possible to predict some aspects of messages under certain circumstances, given sufficient information about the system, e.g., a minimum arrival time.

The concept of predictability arises when dealing with systems having loops or cycles. If each process in the cycle sends a message at time $t$ that is dependent upon a message it receives at time $t$, then each process is a function of itself and the physical system it models exhibits race conditions. The author requires that in every cycle and for every time $t$, there is at least one $\mathrm{pp}$ whose output messages along the cycle can be determined beyond $t$, i.e., up to $t+\epsilon$, for some $\epsilon>0$, given a set of input message up to $t$. That is, at least one $p p$ in the cycle introduces a delay within the cycle. We agree with the author that this assumption is reasonable.

The simulation of the physical system is performed by a simulator, or logical system. consisting of logical processes (lp's). There must be a 1-to-1 correspondence betreen messages transmitted in the logical system and in the real system such that messages and corresponding times agree. Note two things: First, the time ordering of the messages in the logical system and the ordering specified by the real time, at which the messages are processed, need not agree. Second, as will be found later, the logical system may send messages that do not have a correspondence with messages in the real system and are present for purposes of avoiding or detecting deadlock. These messages are not considered in the above 1-to-1 correspondence.

Let $(t, m)$ represent a message with content $m$ and time $t$. The logical system must be able to determine the exact chronological sequence in which message transmissions take place in the physical system; therefore, determining one message and then an earlier message is not acceptable.

The objective of the simulation is to determine the behavior of the physical system by using asynchronous logical components. The processing times and message transmission times should not be required to correspond to those of the physical system or necessarily to the order in which events occur in the physical system, if those events are independent.

Misra first considers a distributed simulation scheme which can result in deadlock. The distributed system consists of a finite number of processes and directed channels interconnecting them. Logical processes, Ip's, execute sequential simulation code and send and receive commands. The send command deposits a messagc on the named outgoing channel and is allowed to continue executing cole. Messarges sent along a channel must be delivered in the sequence in which they are sent. An lp desiring to receive a message from a channel may have to wait until one arrives along that channel.

Definition: A set, D, of lp's is deadlocked at some point in the computation if

1. every $1 \mathrm{p}$ in $\mathrm{D}$ is either waiting to receive a message or is terminated,

2. At least one $\mathrm{lp}$ in $\mathrm{D}$ is waiting to receive, and

3. if $L P_{i}$ is in $\mathrm{D}$ and is waiting to receive from $L P_{j}$, then $L P_{j}$ is also in D and there is no message in transit from $L P_{j}$ to $L P_{i}$.

Associate one Ip with each pp to simulate it. Also connect a channel from $L P_{i}$ to $L P_{j}$ if $P P_{i}$ can send messages to $P P_{j}$. 
If an ip knows the initial state and all messages corresponding to those received by the corresponding pp up to time $t$, then it can simulate the actions of the pp up to time $t$ It may be able to simulate it beyond time $t$. Note: it is the ability to predict beyond time $t$ that may allow some message passing (NULL messages) to be eliminated.

The chronology requirement states the order in which messages are sent from $L P_{i}$ to $L P_{j}$ must agree with the timestamp order in these messages. The implication is that if a message $(t, m)$ is received then the receiver knows all messages hetween corresponding pp's up to and including time $t$ and can simulate up to time t.

Definition: the channel clock value of a channel is the $t$-component of the last message reccived along that channel, and the initial value is 0 .

$L P_{\text {; }}$ knows all messages received by the corresponding $P P_{i}$ up to time

$$
T_{i}=\frac{\min }{j}\left\{t_{j}\right\}
$$

where $t_{j}$ stands for the channel clock value of the channel from $L P_{j}, T_{i}$ is the clock value of $L P_{i}$, and $L P_{i}$ can safely simulate up to $T_{i} . L P_{i}$ sends any messages that the corresponding $P P_{i}$ would send.

The algorithm is as follows:

1. Initialize all $T_{i}$ to 0 .

2. While simulation not yet complete, simulate $P P_{i}$ up to $T_{i}$ by

a. Computing the sequence of outgoing messages for each channel and sending them in sequence.

b. Receiving messages and updating $T_{i}$ until $T_{i}$ changes.

(1) Let $T_{i}{ }^{\prime}=T_{i}$

(2) While $T_{i}^{\prime}=T_{i}$, wait to receive messages along all incoming channels. Lipon receipt of a message, update the internal state and recompute $T_{i}$.

Note that sources only send messages and sinks only receive them. Correctness of the simulation requires a one-to-one correspondence between messages in the physical system and the simulation. Note, however, that it becomes necessary to have messages in the simulation that do not have a correspondence with messages in the physical system. In particular, messages must be sent in the simulation to allow for termination. A simulation is correct iff. every lp has sent correct out ut sequences along every outgoing channel.

A simulation that follows the above algorithm can deadlock. The deadlock can arise because an LP doesn't know that a source has finished and will send no more messages and because of loops in the system, with each lp waiting on its predecessor in the loop.

Definition: The clock value of the simulation is the minimum of all lp clock values. The phrsical system has been simulated up to time $T$, with some lp's having simulated beyond that point. $T$ may be thought of as a global time for the simulation, but this global time is not used. That is, there is no global control.

Misra considers a number of methods of deadlock resolution, which might be termed cleadlock avoidance and deadlock detection followed by extrication from deadlock. 
If $\mathrm{T}$ remains at some fixed value forever, deadlock has occurred and some means must be found for advancing the simulation. The cause of stoppage can occuly at an 15 that has more than one injut and will not receive any more mesaiges along some particular channel. One scheme for avoiding deadlock is to use the (oncept of a NLLL message. (t.mull). A NULL message is a message sent by onc |, to another to indicate that no message will be sent to it between the curent channel clock ralue and the t-component of the message. They have no countorpart in the phrsical system and announce the absence of messages.

To talie adrantage of NLL messages, $L P_{i}$ must be able to predict that after transmission of a message sequence to $L P_{j}, P P_{i}$ will not send any more messages to $P P_{j}$ until time $t_{j}$. Then $L P_{i}$ sends $\left(t_{j}, n u l l\right)$ to $L P_{j}$ after that sequence. If $L P_{i}$ is presently simulating at time $T_{i}$, then each outgoing channel is sent a last message with a time component greater than or equal $T_{i}$, some of which may be NULL messages. But only the last message along a channel in any iteration may be a NULL message.

A NULL message is treated in the same way as any other message, i.e., the lp updates its internal state, including clock value, and possibly sends messages.

In order to simulate up to time $T$, every source must send messages until the 1 -component of the last message equals $T$, even if that means sending a NULL message with time $T$.

The use of NULL messages creates a deadlock-free system which can simulate the given physical system up to any time $T$, assuming the predictability property is met.

Misia makes the point that performance should be judged on turnaround time. not processor utilization. Another factor of interest is line bandwidth. He suggests that for acyclic networks, the above scheme is efficient.

Efficiency is affected by a number of factors, which are

1. The amount of branching - NULI messages tend to be created at branches and may proliferate. The fewer the number of branches, the better the performance. Loops tend to reduce the efficiency.

2. Time-out mechanisms - Since NULL messages have no effect if followed by another message having a greater timestamp, NULL messages might be delayed for a short time before sending them in the hopes a real message might arrive over the channel. The time to wait is a compromise between long deadlocks and increased NULL messages.

3. Buffering - The number of buffers affects performance. The use of few buffers causes the sender to have to wait at times. The use of a large number of buffers causes memory space to go unused at times. Experience shows that adding buffers reaches a point of diminishing returns.

An alternative to sending NULL messages whenever a process becomes inactive is to set a time-out and request an advance of the clock from a predecessor when the timeout occurs. If the predecessor's clock value is greater than the channel clock value, the predecessor can send a NLLL message. to adrance the channel clock value. If the predecessor camnot adrance the clock value and is idle, the predecessor can request certain of its predecessors to advance the corresponding channel clock value.

The lp requests by sending a query message. Since query message are likely to be propagated. they will contain the path which is traversed and the channel clock values of all channels along the path. A reply 
contains the query and a new clock value for the last channel in the query, where this last channel is able to increase a channel clock value. The author supplies a set of rules for the actions of an lp initiating a query or reply and for receiving a query or reply. These rules will not be presented here. Note that if a query is received by the lp initiating the query, a deadlock has been detected. Thus, the query either results in increasing the channel clock value or in the detection of deadlock.

An lp that detects deadlock is not necessarily able to resolve the deadlock. Resolution is accomplished by determining the minimum value of the "next event times." The "next event time" for an lp is that at which a message will be sent by the lp provided no further message is received up to then. For a deadlocked set, the minimum of such times is the lime to which that lp can be advanced. One method of determining this time is to include the minimum of the "next event times" and the associated $\mathrm{lp}$ for the set of lp's encountered by the query message. The relly from the processol that detects deadlock can then indicate the processor that is to restart at that clock value.

Yet another method is the use of a marker. In this scheme, the simulation is run until deadlock, deadlock is detected, and recovery from it is initiated. A marker circulates through the network in such a way that it traverses every channel of the network during a cycle. If the network is not strongly connected, channels can be added to make it so. The marker, a special type of message, is initiated at some lp. The obligation of an lp receiving a marker is to send it along the designated path within a finite time of becoming idle. The marker carries information needed to detect deadlock as indicated above. When an lp becomes idle and sends on a marker, it sets a flag so indicating, but then resets it if it becomes active before the marker returns. If the last $\mathrm{N}$ lp's, where $N$ is the number of:Ip's, visited by the marker have their flag set, then deadlock has been detected.

The marker keeps a count of the number of lp's it has seen with flags set since the last $\mathrm{lp}$ with the flag reset. It also carries the minimum of the "next event times" for the lps in that count. When deadlock is detected, the minimum time and the associated lp are known. When the marker reaches the lp, that lp can restart using the new channel time. Again a tradeoff exists between slow marker movement, with slow deadlock detection and response, and fast marker movement, with a high number of overhead messages. The schemes can be modified to detect deadlocks within a subnetwork, where the subnetworks are determined through preprocessing. Misra does not discuss the preprocessing.

Misra suggests that empirical investigations need to be carried out on rarious hemisticin to deremine

1. which heuristics work well for which problems on which machine architectures

2. how to partition the physical system among a fixed set of processors, and

3. how to set simulation parameters such as time outs and buffer sizes. 


\subsubsection{Bagrodia, Chandy, and Misra.}

In the approach taken by Bagrodia, Chandy, and Misra [12] a physical system is modelled by a set of message-communicating processes. Events are modeled by message communications. An entity is a process operating in simulated time. A message-based simulation language, MLJ, which is in the public domain, is presented in the paper.

The authors propose three primitives for message-based simulations:

1. CREATE and TERNIIATE processes

2. SEND messages to processes

3. M-t]T for mesages and/or simulation time to elapse.

The advantage of messige-based simulation lies in its use for simulating distributed stems. (And in distributed simulation, one would assume.)

A system being simulated is assumed to consist of a number of physical processes which interact at discrete instants of time. The interactions are called events; the time instants, event times, and the simulation of the physical process, a logical process. An event is the communication of a message. A request message requests service of the process; a reply message is a reply to a request message.

A simulator clock is used to represent the passage of time in a simulation. The simulator clock advances in discrete steps, where each step simulates the passage of me between two events in the system. The authors allow for a process to wait until a time $t$ for a specific type of message. While waiting, it may buffer other received messages. Thus, the following primitive is proposed:

\section{WAIT $t$ for $b$}

where $\mathrm{t}$ is an integer-valued expression and $\mathrm{b}$ is a Boolean condition. Specifically, $b$ is used to specify the messages that the process is ready to accept and $t$ specifies the maximum time up to which the process is willing to wait for the message(s). The process ceases to wait either when the simulator clock reaches the value represented by $t$ or if it receives a message that satisfies the condition $b$, whichever occurs first. Messages may be given a type designation, e.g., emergency. When the simulation clock has advanced to $t$, the process has timed out, and a timeout message is sent to the process from the simulation monitor. The process then proceeds to examine the input queue for messages to process.

The authors distinguish two basic building blocks, resources and processes, where a resource is a passive object and a process is an independent, dynamic entity. Processes use resources to achieve an objective. One questions the representation of a resource as a passive object, defined as a simple variable or a complex data structure. However. the authors then recognize that the difference between the two is relative, and they propose the concept of an entity, which seems reasonable. Situations may exist at which it is advantageous to look at an entity as a resource or as a process.

An entity is a sequential program module implemented in the host language with the capability to 
1. Create other entities.

2. Terminate itself.

3. Send messages 10 other entities.

1. Nait for the passige of time and/or receipt of messages.

An entity-type represents a class of object; an instance of an entitytype represents a specific object of its class. An entity is an instance of an entity-type. CREATE allows multiple, independent instances of an entity-type to be dynamically created. One starts with the entity MAIN, which initiates and terminates simulation. An entity may terminate itself by executing the END-ENTITY statement, and cannot be terminated by another entity in the system.

Entities are in one of the two states, active (executing) and waiting (inactive). An entity moves to the active state either when it receives a message satisfying the condition specified by the WAIT primitive or if the time specified in the WAIT primitive has expired.

Messages are buffered. Specifically, messages which are sent are deposited in a FIFO manner in a message buffer associated with the recipient entity. If an entity enters a wait state, entries in the buffer are examined for one of the type specified in the condition. If one or more such messages are present, the first one in the buffer is received, the entity enters the active state, and the message is acted upon. Since received messages are accepted and retrieved in FIFO order, it appears that messages could be executed out of simulation time order.

Communication of messages takes 0 simulation time. However, delays corresponding to delays in the real system can be modelled as a delay at either the sending or receiving end.

Entity types consist of an entity heading, a local variable declaration section, a message declaration section, and an entity body. An entity-type may only have input parameters. In the body of the entity, the following statements may appear:

1. LET - used to create new entities

2. END-ENTITY - used to terminate the entity

3. INVOILE - used to send messages

4. WAIT - used to wait as discussed above

These are defined as follows:

LET - Every entity is assigned a unique ID stored in a variable of type entity-identifier. A LET statement creates a new instance of the slecified entity type. A newly created entity obtains its ID through the valiable myid.

ENT)-ENTJTS - Causes hoc (ntity to be terminated.

MTOKE - The INTOKE statement is of the form

NWOlEE el with ml(parameter list) 
where el is an entity. ID. The message of type $\mathrm{m} 1$ is then sent to el prorirled el exists. an $m 1$ message has been declared for entity el, and formal and actual parameters of message $\mathrm{ml}$ match. The authors state that "An attempt is made 10 deliver the message at the current value of the simulation clock." The meaning is not clear, and the subsequent sentence is garbled.

MAIT - AS discussed previously. "Condition" may be "true" or "false." Also. the time may be infinity, corresponding to being in the waiting state when not busy.

The authors give a model message-based simulation of a doctor's office. In this model, doctor, receptionist, and patient are modeled as entities. The model does not contain complicating factors such as multiple doctors and loops such as the patient going back to the receptionist to pay the bill.

The capabilities of the simulation system as described in the paper appear to be very limited and of not much use for DDES. Selection of messages based on condition is not sufficient to allow the creation of a DDES where simulation must progress based on simulation time. Also, the simulation clock discussed in the paper appears to be a global clock, which again is not appropriate for DDES. The specification of conditions for choosing messages is an advantage, as is the ability to dynamically create and terminate entities. The concept of deadlock is not even mentioned by the authors. 


\subsection{Nethods lnowing Rollhack}

The second type of DDES method involves continued processing even though procesing may be in enror. If a task is processed out of order, a rollback oceurs to a brejously known state which was correct. Processing resumes from that point. The inventor of this scheme is Jefferson.

\subsubsection{Jefferson}

Jefferson [13] introduces the concept of virtual time for distributed computation and implements it in the Time Warp (TW) mechanism (an operating system). Virtual time may be used for synchronization of distributed simulation and as a concurrency control for databases.

TW relies on general lookahead/rollback for synchronization. Possible synchronization conflicts are ignored until discovered, and then a rollback occurs to before the conflict. Then reexecution occurs along the new path.

The author clains that:

1. Distributed deadlock has a natural and elegant implementation.

2. Even if rollhark occurs. time would have been wasted by the processor any ray in of her implementations.

3. Rollback is relatively infrequent. (conjecture.)

The author presents some definitions which are repeated here.

Definition: a virtual time system is a distributed system executing with an imaginary virtual clock reading virtual time. Virtual time is a global, onedimensional, temporal coordinate system and is used to measure computational progress and to define synchronization. It is totally ordered. Virtual time is implemented using many local virtual clocks, loosely synchronized. Clocks may occasionally "jump backward," but tend to go forward.

Definition: An event is a set of actions that take place within a process $x$ at virtual time $t$, signified by $(x, t)$.

Processes communicate by sending messages but there need not be a specified channel between them, as long as there is at least one path for the message transmission.

All messages contain the information:

1. Name of sender

2. Virtual time sent (virtual time at moment message is sent)

3. Name of receiver

1. Virtual receive time (virtual time when message must be receivel.)

The fundamental Pules for Tirtual time syatems are:

Rule 1. Tirtunl send time must be loss than virtual receive time.

Pule 2. Erents must he processed in rirtual time order.

The abore are Lamport's Clock Conditions. All messages output are in rirtual 
time order, but not necessarily in virtual receive time order. Messages input to any one process are read in virtual receive time order, but not necessarily in rirtial send time order.

Definition: An event at $(x, t)$ is an ordinary deterministic sequential computation conducted entirely within process $x$ and involves zero or more of the following:

1. a may receive and read any number of messages intended for it.

2. read the virtial clock

3. uplate state rariables

4. send any mumber of messages.

$A$ constraint is that if an erent $A$ causes event $B$, then the execulion of $A$ and $B$ must be scheduled in real time so that $A$ is completed before $B$ starts. (A (anses $B$ means A occurs before $B$ and has an influence on $B$.)

If no causal relationship exists between $A$ and $B$, then the actual order in which $A$ and $B$ are scheduled is arbitrary.

In the paper. Jefferson compares his work to the work of Lamport, Reed, and Schneider. but this comparison will not be discussed here.

Definition: A timestamp is the virtual receive time of a message. Messages must be processed in timestamp order. In the approach taken by Jefferson. messages cannot be guaranteed to arrive in timestamp order. The TW mechanism must solve this problem.

TM contains a local control mechanism and a global control mechanism. Local control is concerned with assuring execution of events in receive time order; global control is concerned with global issues such as $1 / O$ handling.

The local virtual clock' at each process only changes between events and changes to the value of the the timestamp of the message with the lowest timestamp in the single input queue. When a message is received out of timestamp order, rollback must occur to an earlier virtual time, and forward execution must begin again. Lack of messages means termination (setting of the local clock to tinf), at least temporarily.

Each process excutes continuously, processing those messages it has reccived in virtual time ordcr. If a message comes in with a timestamp lower than the present virtual lime. the process must roll back to the virtual time specified by the timestamp of the message or possibly a time before that message. Rollbaci: elicits a penalt $\%$

"Time Narp" refers to the fact that the rirtual clocks of different processes do not agree. and in fact. time may occasionally go backward. Rollback is made transparent to the programmer. Where more than one process executes on one processor, scheduling is accomplished by always running that process whose virtual time is farthest behind. Although dynamic reassignment could occur. the author assumes that it does not.

In a distributed environment, rollback is complicated by the fact that messages may have been sent to other processes, which in turn may have sent their own messages. and so on. Some or all of these messages may be wrong, because they were processed with an out-or-order timestamp. Some of these messages may have requested output. which may be in error. These incorrect messages must be "unsent" and side eflects. reversed. These are handled by the time warp system. 
A process is considered to be composed of

1. A unique process name.

2. A local rirtual clock.

3. A state. which is the entire data space of the process.

4. A state gucure combining sarul copicm of the process recent states at

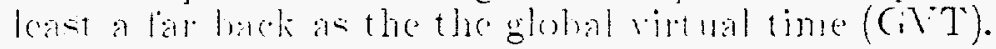

5. An infut qune. comtaining all recenly arrived messages sorted by virthal lime. al least as far back as cill.

6. An ontpur queuc. containing negative copies of processed messages in virtual send time order, at least as far back as GVT.

An antimessage is identical to the original message except for its sign, which is minus. A process sends a positive message to another process and retains a negative copy in the sender's output queue. Whenever a message and an antimessage occur in the same queue, they annihilate one another. A negative message could arrive first.

As stated earlier, when a message arrives at a process with timestamp earlier than the present virtual time, rollback occurs. The first step is to search the state quene for a state of the process before the timestamp of the message and 10 restore it. All states sared after that time can be discarded. The process is started executing at the time of the sared state. Next, messages with rirtual senrl times greater thau the rollback time are unsent by sending their antimessages.

What happens at the recejving end of an antimessage depends upon the timing. If the original message has arrived but has not been processed, the antimessage will annihilate with it. leaving no record. If the original message has been or is being processed, the receipt of the antimessage will cause rollback to the timestamp of the positive message. Positive and negative messages will annihilate. Processing will proceed, starting with the message having the earliest timestamp, and antimessages may be sent. Finally, if the negative message arrives before the positive message, it will be enqueued and will annihilate with the positive message when it arrives. If it is selected for execution because of its timestamp, the author suggests that it be treated as a no-op. When the positive message is received. annihilation occurs, but rollback does not.

The authors claim that deadlock is not possible, because no blocking occurs. Also, the amount of rollback is limited, with the worst case that all processes roll back to the same virtual time as the one that originated the rollback.

The author uses the concept of the temporal locality principle to suggest that in a realistic system, rollback is not as costly on the average as might be feared. The temporal locality principle states that most messages arrive in the rirtual future at their destination and do not cause rollback, and that those that arrive in the past, arrive in the recent past.

The cost of virtual time synchronization is only the cost of rollback and antimessage orerhead. Note that the time used for computation of messages. which are erentually molled back, should not be considered because the only alternative is to block and not do any computing.

The global control mechanism consists of GVT, which at real time $r$ is the minimum of (1) all rirtual times in all riptual clocks and (2) of the virtual send times of all message that have been sent but not yet been processed. 


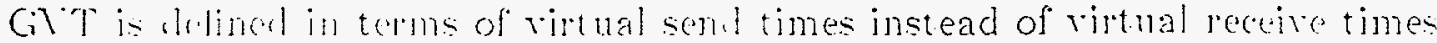
because of the Hom control protocol which is discussed later in the paper. (6) T never decrenses and acts as a floor on virtual times to which processes may erer again roll back. Proviled some realistic assumptions hold, GVT must eventually increase. Any event with a rirtual time less than GTT cannot be rolled back and is irrevocably committed with safety.

The definition of GVT is not practical. Although user processes do not need GVT. it is used for global control of the system. From a practical point of view. GVT is less than or equal to

1. all virtual times in all virtual clocks in the "snapshot," an instantaneons picture of the system taken at real time $r$.

2. all virtual send times of messages that have been sent but not yet acknowlerged (and may be in transit).

3. all virtual send times of message in input queues that have not yet been processed.

The mastor process hroadcasts a recuest to each process to provide it with the

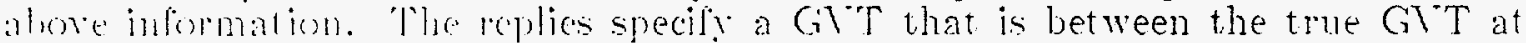
the moment the reguest is sent and that at the moment the determination is completed. This slightly outdated time is generally acceptable.

GVT is estimated with some frequency and is generally a compromise ralue. Higher frequency allows fast response time with better space utilization, but with increased processing time and increased usage of network bandwidth.

Two uses of GVT are in memory management and flow control. The authr claims that memory management algorithms are simple and natural. In addjtional to the normal usages of memory, memory is required to store 1) old states. 2) messages in output quenes, 3) past (processed) messages, and 4) future (mprocessed) messages. The first three support rollback and are managed similarly, i.e.. if the virtual receive time of a message is greater than GVT, it is discarded and al] of the saved states with time older than GVT can be discarded.

The management of unprocessed messages is a control flow problem. Since such messages must be buffered, a point may be reached where the receiver's buffer is full. One possibility is to return the message with the latest receive time to the sender. If the sender can accept it, it rolls back to the state prior to sending the message and restarts from that point.

In TW, detection of termination is handled in terms of GVT. A process sets its local clock to tinf whenever it runs out of messages to process. If GTT is tinf, all processes have terminated and so does the simulation.

Processes committing a run-time error are marked as being in an error state.

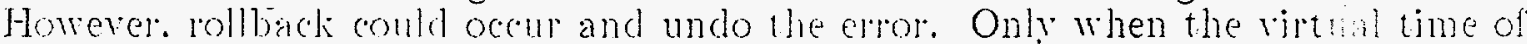
the procesis annot he rollod hack to a time lefore the error is the errol considered to be aretual and be repereled.

I/O ojerations must be held until such a time as rollback cannot take place and undo the operation. Thus. the $I / O$ operation is performed only when GT $T$ exceeds the rirtual receive time of the $1 / O$ command.

Should a "crash" occur, the preferable approach is to restart at some intermediate point rather than at the beginning. Determining a consistent state of the system for restarting a distributed system is more difficult than for one that is not. A snapshot. i.e.. state, of a virtual time system at a particular virtual time $t$ can be constructed by having each process take a snapshot of itself as it passes 
virtual time $t$ in the forward direction (and unsnapshots itself whenerer it rolls back over rirtual time $t$. Whenever GT exceeds time $t$, the snapshot is complete and ralid. here.

The paper finishes with a number of examples which will not be included

\subsubsection{Berry and Jefferson}

The paper by Bery and Jefferson $[1-1\}$ discusses the concept of object-oriented simulation and gives a method for calculating nontrivial lower bounds on the time it takes to execrite such a simulation in parallel. The method is based on a criti(a) path analysis.

Exchange of information is ria messages. The erent queue is dist ributed. and each object has ils own simulation clock.

The described method measures the potential concurency arailable in simulations. Two possible uses are 1) to apply it to a particular simulation proman to see how much concmuency is available and to use bot leneck analysis to inprove its potential peromance in a distributed environment. and 2 ) to evaluatc a distributed simulation mechanism by running benchmark simnlations under then for comprarison of the actual with the anticipated concurrency.

Procedure:

1. Instrument a sequential execution of the simulation for a time trace.

2. Transform the trace into a directed graph whose edges are weighted with the execution or communication times expected for the hardware on which the distributed simulation will run.

3. Apply a critical path algorithm to find the longest weighted path.

The path so determined constitutes a lower bound on the execution time for the simulation on the specified hardware. Bottleneck objects are determined by determining the ratio of each object's execution time to the entire system's completion time. Those objects with the highest ratio are candidates for performance optimization.

The havdware is assumed to consist of identical processors whose message communication oredhead plus transmission delay between any two processors is contanu. Civen are an object-oriented program, $S$, the input for a particular execution of S. and the processor speed and communication delay constant.

Sequential constraints are

1. If two events are scheduled for the same object with simulation times $t_{1}$ and $t_{2}$ with $t_{1}<t_{2}$, then the first must be executed before the second.

2. If one erent causes another to be scheduled at a later time. then the one. must be scheduled before the other.

These rules determine a partial order of the erents in a simulation.

The trace determines 1) the name of the sender, 2) simulation time when sent. 3) the name of the receiver, 4) the simulation time at which it was schednled to be receiverl. The trace is converted to a directed graph. which can used to dernce the lowe bound on execution time. An algorithm. such as PERT. can be 
used to find the critical path and its weight. The running time is linear in the number of nodes plus arcs. One can vary such factors as message time to analyze the effect of message times on the outcome. One conclusion reached is that communication time contribues to the amonnt of inherent secuentiality, but does not account for all ol it.

\subsubsection{Soliol. Laskomiti. and Nugent}

The paper by sokol. laskowski. and Nugen [15] addresses jtself to one aspect of DDES using Jeflerson method. i.e. the assignment of processes to proccsors. The olject behariors are in the form of IF-THEN rules which list the actions to be taken by an object on receipt of particular messages. The paper categorizes oljects as either basic or auxiliary. Basic objects are actors in the simulation and represent some real-world object. Auxiliary objects assist in the construction and conduct of the simulation.

The authors speak of a prototype based on the Cal Tech hyercube, withoun shared memory; communication is through message passing. Time Warp would be the mechanism for implementing the simulations.

These authors, as well as others, emphasize that the degree of achievable concurrency is directly proportional to the degree of physical concurrency in the model.

Two aspects of Time Warp simulation are the assignment of information and processes to objects and the placement of objects on processors. Constraints on allocation include the size of memory and the speed of communications.

Strategies for allocating objects to processors include:

1. Place all objects on one processor - result is a slow serial implementation.

2. Place each object on its own processor - the number of available processors places a limit on this method.

3. Place several objects on one processor - Inter-object and inter-processor communications play a role in how this assignment is performed.

Strategies for allocating information to objects include:

1. Give an object all pertinent information - a high degree of replication may result.

2. Give the object most of its frequently used information - additional information is obtained through queries.

3. Assign objects a minimum of information - results in minimal synchronization costs but high waiting times.

The implementation strategy should be to minimize the execution tinu. detined as the time elapsed from when the first processor begins until the last 1rocesol terminates. Assumptions are that processor memory is not a limitation aud that communication speed is reasonable rotive to the application.

Execution time consists of procesimg lime communication time (transmi-ion

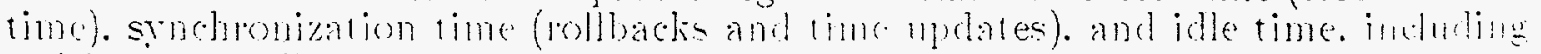
wating for replim to queries. 
The tyes of message are guery. mulate and action. Querjes do not ause molhack but update and action mesages may.

The authors discuss ways of optimizing the execution time. One suggestion is to place an object having frequent rollhacks onto more than one process as a form of load balancing. Doing so could create as many problems as it is intended to solve. A synchronization problem could result.

Pollback can he kejt. low by minimizing the virtual time difference between sender and receiver. The objective is to have the processors run at the same simulation speed. The way in which objects are assigned to processors has a bearing on this matter. It may le possible to partition objects, so that operations associated with an object are performed in parallel, thus reducing the time for the object to produce its result.

The authors see the need for auxiliary objects, which could be collections of related functions (procedures) or related objects (databases). When many objects attempt to use these auxiliary objects, they can become bottlenecks, and thus require careful consideration. Where necessary, the procedural objects could be replicated in the objects where they are needed.

The database objects constitute a more complex problem. Those portions of the dat a base that change infrequently can be replicated. where necessary. The update communication costs would be minimized in this way. For a large database that changes frequently, one approach is to partition the database among processors. in order to decrease the waiting time.

The authors see the way of increasing the speed of simulation for a particular application to be an iterative process. Based on run time data, the user can alter the way that objects and information are assigned to processors in order to optimize the results. (Another altemative would be to introduce dynamic reallocation of objects, either between simulations or during long simulations.) 


\subsection{Methouls Involring Both of the Previous Schemes}

Each of the previous schemes has its drawbacks. One thought is to try to combine the two methods. taling the best parts of both. That is what the next author at tempts to do.

\subsubsection{Chlamtac}

The paper by Chlamtac [16] appeared interesting because of its claim that the method combines a number of DDES techniques, including Misra/Chandy and Jefierson trpes.

In the proposed method. a processor is allowed to actively seek information "for maximizing the probability of correct operation." It is also allowed to contimue operation on the basis of only partial information, in which case corrective action may be repuested. The algorithm is responsible for optimizing performance by continuously lanancing state correction penalties against forced delays. Algorithms do not store observations, in order to reduce the computational penalty associated with corrective actions with respect to data collection and analysis of stat istics.

The system consists of physical processes, pp's, and logical processes, lp's. with lp's assigned to pp's. Messages are pairs $(t, m)$, where $m$ is the message and $t$ is the sending time. Messages sent from a process must be in non-decreasing time order. The next message chosen for execution is determined by the minimal time from the collection of messages present on incoming links. A processor may continue with only partial information on incoming links. As in Jefferson, the last known correct state is recorded. Periodic update of correct state uses a "negative acknowledgment" mechanism, where the period depends upon the amount of available storage and the cost of communication. Execution in the presence of partial information does not necessarily lead to incorrect operation; therefore, system operation is optimized to minimize local simulation time by balancing the cost of state correction against the enforced delays required by a strictly correct execution of the model.

Assumptions: A single storage buffer exists at the receiving end for each link. The receiving processor must accept or process the message before another can be sent over the link. Each processor has a local time set to the time of the last messige it accepted and has times associated with each link to it, which is set to the time of the last message sent over e link and residing in the buffer.

The author distinguishes between the operation of an $1 p$ when it has only one incoming link as opposed to having more than one. Note that there is CONFL:SION of notation, since $m$ is used to denote a message, a message number. and the number of links into $L P_{i}$.

Single link case: If the buffer contains a message with a time greater than or erual to the local time. update the local clock. free the buffer (seems too soon to free the bufler). attach a record to present list that includes 1) message number. 2) message time. 3) and statistics collected "under current message arrival". Partly because of the confusion in the use of $m$. the paper is not clear as to whether the message itself is retained. From here, the algorithm goes to the common part discussed below.

If the local time is greater than the message time, the local statistics are corrected as follow's: Consult the list of records and accept the statistics associated with the message haring the largest time less than the present message time. (The latter is an interpretation of what the author means by "first" message). 
The author is not clear on this point, but it appears that one again goes to the common part, since this new message must be sent. The author says that the other messages need not be resent. Indeed, that is unwise because one can expect more messages to be out of order. What if, as a result of an out of place message. a previous message should not have been sent?

Note: The authon is recommending that running totals of statistics, such as mein. variance. and percentiles. be kept, obviating the need to store observations from the beginning of the simulation, but only from the time associated with the last correct state inslance. At this point in the paper. the author has not yet rillied aboul hom llat time is determined.

Multiple incoming links case: The lp computes the minimum time over all messages in occupied buffers. If the buffer contains a message with a time greater than or equal to the local time, update the local clock, free buffer (seems too soon to free the buffer), attach a record to present list that includes 1) message number. 2) message time. 3) incoming link, and 4) statistics collected "under current message arrival". Here again, the message may also be stored. From here the algorithm goes to the common part discussed below.

If the buffer contains a message with a time less than the local time, corrective action must be taken by resending all messages with time greater than the time of this message. (Note that the p.t $=$ ti case is not considered; Nor is the case $\mathrm{ti}=\mathrm{Ti}$ considered.) (This step is not clear at all.) The lp computes the minimum time over all messages in occupied buffers. (If the first time through this step, then you have the value. It makes no sense to recompute the minimum time unless the buffers can be changed while this step is being performed.) The procedure fails to state that one first consults the list of records to find the message associated with the largest time less the present message time and accept the statistics associated with it. The next message to be processed is the one just received that caused the "rollback." That is not clear from the algorithm. nor is it clear what is done with the message. Does it overwite a prevjous message in the list? Does it simply get inserted in the list in its proper place. and if so when? Now point to the next message in the message list. update the local clock, recompute statistics, send the message, move the pointer to the next message, and repeat until the stored messages are exhausted. If the message time of the next message exceeds that of the minimum message time in the occupied buffers. then a new message with lower message time has arrived, and the correction process must, be started over again from this new message.

Common part: Compute the message (event) processing time, $T_{e}$. Prepare to send a new message with time equal to present time plus $T_{e}$. Before sending the message on link $(i, j)$, check the message buffer $C_{i j}$ for empty. If empty, send the message to the bufier and set the buffer to indicate occupied. If the buffer is occupied and the time of the present message is less than that of the message already in the buffer, then overwite the message with the new message. Otherwise wait until the buffer becomes empty, and then send the message. Once the message is sent, start over. It would seem to be better to allow the process to continue computing, if it can, and be interrupted when the buffer becomes empty.

The author gives an example. Howerer. the example does not include all the intricacies that could occur.

At this point. the author makes clear that messages are actually stored, not just the message number. The author proposes an approach in which an lp (an) request retransmission from a specific. logically preceding lp. Let

$T=T_{j} \mid T_{j}$ is the lorat time of logical process $L P_{j}$ and a path exists $L P_{j} L P_{i}$.

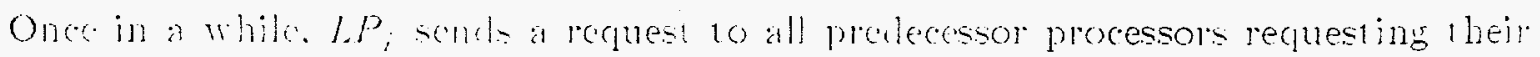


local time. It then detemines the minimum and uses this time to determine what to ematrom its local momory in terms of past messages and associated statistics. Indete if all predecesoms are requested to supply the information. then $\mathrm{T}$ is the minimum time that he procesor will see in the future. Note that the number of merages can be jeduced if. when a processor must relay a message, it does not send one of its own. but instead waits until a reply is received. determines the minimum of its time and the time in the message and sends the minimum of the two. Thus. it appears that by being selective and setting up a specific route, the number of recuest messages and replies can be reduced. The author suggests that the intermediate nodes can also use the information. That appears to be true if there is no loop. but does not appear to be true if a loop exists. Note that in the suggested method. the routing information must be included, so that the intermediate nodes can include their local time in the evaluation.

Variations exist on the procedure. For example, in a loop situation, an $1 \mathrm{p}$ could send a request message to its successor with a request that the message be routed through the remaining processors in the loop. This step could still require a significant amount of effort. The author does not indicate what happens if memory is full and the process is unable to erase any messages because the time received back will not allow it. It looks like a deadlock that is resource based conld be created.

The author atcempts to show that 1) Deadlock does not occur. 2) Termination occurs. In order that termination oceur. it must be shown that global and focal simulation time increase contimumsly and all message up to time 1 are correct. The author gives no proof. Note that rollback does occur; therefore, we are saying that over the long haul. the times increase.

The author proves the following theorem: Given node (LP) processing times are nonzero, no deadlock will result in the system. The proof is similar to that given by Misra. He attempts to show that if a loop exists, times from one processor to the next must be the same or increasing around the loop, that the inequalities can only be satisfied if they are equalities, and that nonzero processing time results in a contradiction.

Problem: It appears that the only restoration that occurs is with the statistics, and tha: there is no effort to restore the proper state at that time. 


\subsection{Methods Involving Data Flow Concepts}

The message passing that takes place within all these DDES methods suggests that the use of a data flow concept may be advantageous. That is what the next author attemprs to do.

\subsubsection{Jayaraman}

Jayaraman's [17] objective is to apply dataffow concepts to the DDES problem. In his presentation. the author assumes that data is time tagged. This time tag rejpesents "the local time of some temporary entity in the simulation model." In the barbershop problem. for example. the entity is a customer at some stage of the process. and the data is information related to the customer. The processing of the data is an erent. There is no ghohal clock or central schechuler in the dataflow model.

In addition to the processes themselves. the author introduces three primitives: delay, choice. and merge. These are discussed next.

The first primitive is delay. On the D input of the delay unit is a stream of nonnegative interers: on the $S$ input is a time-tagged stream of information. The time tag for the exiting stream, output S', adds a delay taken from input D. However. the time to which the delay is added is the max $\left(t^{\prime}{ }_{i-1}, t_{i}\right)$, that is, the maximum of the previous exit time and the new entrance time. In effect. a new element cannot enter the delay until the previous one has exited. For example, if the cashier in the barbershop problem is modelled as a pure delay, then the cashier cannot process the next customer until the previous one has exited.

The next element is choice. The choice primitive selects the output line to which to send the input data on line S. Line B could an an alternating sequence of 1 's and 0's or be a random sequence of bits. The choice module could easily be extended to have three or more outputs with line B indicating a choice of one of the output lines, much like a demultiplexer. Such a primitive could be used in front of the set of barbers to choose to which barber to send a customer.

The last primitive is merge. It time-sorts the incoming data according to the time tags. Such a primitive could be used in front of the cashier in the barbershop problem. It, too, could be extended to include three or more inputs.

There are also source and sink operators. In the barbershop problem, the source would generate customers for the shop.

The author presents the idea that deadlock occurs in cyclic dataflow simulation graphs due to circular dependency between the output of a merge operator and its inpul seems basically correct: however, that view seems too simplistic. Assume that there is some way to get started and to pass a data item through the merge operator. That shonld be quite possible. Simply make sure that the merge operator receives a (time tagged) data item at both inputs upon initialization. Then the data item can progress through the system and possibly come back to the merge operator. As long as there is a (time tagged) data item on each input. one of them can pass through the merge operator. The problem arises when there is not a data item at one of the inputs.

Now let us look at the method for breaking deadlocks, which consists of four phases: TEST, START, EXECUTE, and RE-TEST. During TEST, a test message is sent around the cycle by a predetermined merge operator, which polls information from each delay and merge operator. The intent is to determine which merge operator must break the deadlock. The method will be explained later. In the START phase, the merge operator chosen to break the deadlock is 
Sent a START message. In the EXECUTE phase, operations take place on the dat that was releascd by the chosen merge operator. When the data reaches a choice operator. it sencls an exit message around the loop with the intent of determining when the data item leares the loop. (Apparently when a data item leares the loop. it cannot return to the merge operator, which then cannot proceed. moniug deadlock. When deadlock is determined by a choice operator, it sends a BFIEST mescape to the predetermined merge operator to re-initiate the TEST phase. At thi point the guestion arises as to when the TEST message is sent.

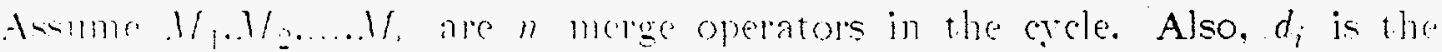
composite detay of all delay operators between $M_{i}$ and $M_{i+1}$. The delay $d_{;}$cannot be calculaicd in advance, since the delays are determined by the $D$ injut. Howerer. for a particular instance of deadlock, the input on the $D$ line can be detemined. A question that might be asked at this point is. "Does the value on the $\mathrm{D}$ input depend upon the time at which the TEST input was received?" The author clefies loci as the time of the composite delay operator. It is not clear how the value of loci is detemined. If it is the time tag of the input to one of the delay elcments. then which delay element, the first or the last? Finally, $t_{3}$ is the time tag of the first data value of the input arc of $M$; that is not in the cycle.

Some problems with the method appear to be that the delays are not a function of the data, and that for a practical problem it should be. For example, in the barbershop problem, the operation performed by the barber depends on the data (task) passed to the barber. There does not appear to be any way to create two data items from one data item with this model, which is also necessary for a practical problem. Since the paper is not clear on what loci is and how it is determined, the paper was not examined any further. 


\section{CONCLUSIONS AND RESEARCH AREAS}

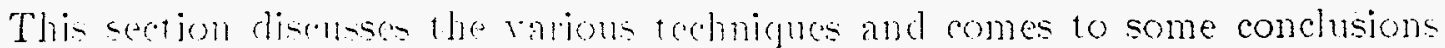

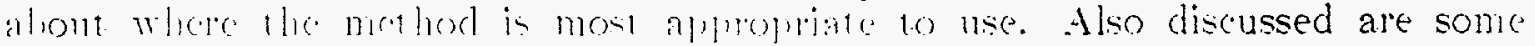
research areats that loxk world pumbing.

\subsection{Concinsions}

All of the methods presented in Section 2 require messages in the logical system that are not present in the phrsical system. In Misra type srstems. one uses XULL messages to allow for termination and to either detect and break deadlock or to aroid deadlock. In Jefferson-type systems, incorrect messages could be sent and the occurrence of these forces antimessages to be sent. The number of these messages that do not correspond to messages in the physical system can become quite large and reduce the efficiency of the simulation.

Misra-type systems are most appropriate under the following conditions:

1. The number of merge-type operators is small.

2. The number of loops is small.

3. The $\epsilon$ referred to in the predictability condition is not small (relatively speaking).

Unless these conditions are met, the number of NULL messages that must be sent becomes 100 large and dradlocli detection or avoidance becomes quite difficult to implement. Furthermore. the implementation of deadlock avoidance or deadlock detecting and breaking is up to the user. No one has proposed an automated process for performing these tasks; that is, they are not transparent to the user.

The Misra-type methods have other drawbacks. Some of these are:

1. Processes cannot be dynamically created and destroyed.

2. Messages sent over a channel must be sent in timestamp order.

3. A number of methods exist for deadlock detection and avoidance. No one method is best for all situations.

4. A process must wait until each of its channels has a message to process. In that way, it can decide which message to process. However, some channels are used frequently and others infrequently.

5. A channel that ends up being unused for a particular set of data inputs does not look like a nonexistent channel, but instead creates significant dificulties and uses significant orerhead to keep the system going.

On the ofler hand. the Jefferson-type method has a number of adrantages. There are:

1. Processes wall be crated and destroyed dynamically.

2. Messages sent orer a channel need not be in timestamp order.

3. Pollback and recovery are transparent to the nser. Of course, an operating syem. such as Time Marp. must be designed and programmed to implement the operations of detection of out-of-sequence messages. saring of correct states. rollback to a previous correct state, and general 
lecorry from meswges that may have been erroneously sent. Jefferson has done that and in principle. that is not diflicult to understand. Rollback is one tsablished technicue in fault-tolerant computing.

4. Only those messages that are known to be incorrect need be resent. along with an antimessage cancelling the incorrect message.

5. The Time Warp mechanism is to some extent self-stabilizing. That is, those processes that have the oldest timestamps are the ones along the critical path and are much less likely to be rolled back. Those which are processing well into the future are likely to be rolled back, but they are ahead anyway.

Jefferson's method also has some disadvantages. Some of these are:

1. The number of erroneous messages and corresponding antimessages that must be sent rulcl berome quite large. In simulations perforned by Jefferson, the percentage of antimessages was significant, $15 \%$ in one case. The latter is with the so-called "lazy" cancellation method. It is larget if "aggressive" cancellation is used. These refer to how soon antimessages are sent after discovering that a message was received out of order. In the "lazy" method, one waits to determine if the message that was sent was correct or not. If it was correct, then an andimessage is sont: otherwise. it is not. In the "aggressive" method, an antimessage is sent immediately.

2. The operating system required to implement the Time Warp concept is in itself a significant undertaking. One either has to adapt Jefferson's Time Warp operating system to his/her own machine and application or write one to do the job. Note that the present $O S$ has a non-portable kernel with a portable executive. Most of the program is written in " $\mathrm{C}$ ", with a small part written in assembly language.

3. The concept of Global Virtual Time (GVT) is necessary from a practical point of view, e.g., for memory management, for determining when $\mathrm{I} / \mathrm{O}$ operations may proceed, and for determining when detected errors, e.g., overflow, are real, and not just a result of messages processed out of sequence. In the case of a large number of processes, the determination and distribution of GVT can be expensive. Howerer, the GVT may be estimated and may lag behind actual GVT.

4 The mothod tends to be memory intensire. e.g. for storing state information and mesages.

5. Some tuning is refuired to optimize the method and to obtain the best operational time. Two such parameters are how often the state is saved and how often Cive is calculated.

6. The operating srstem should he an operating system only that is used for simulation. Jeflerson feels that it should not be implemented on a li $]_{-}$system. for example.

Some discussion of DDES apjeared in the modsim newsletter (E-mail address is "modsin (a (am.unisys.com" or ".sdcrdef'sdcjove!modsim"). Many of the items that appeared in that medium have already been mentioned above. However, a few have not, and these are presented next. Jerry Freedman, Jr., (Sept. 18 correspondence) feels that debugging programs using Jefferson's method would be diflicult in large simulations. That in addition to catching errors in the 
simulation. it would be necessary to catch bugs in the rollback support and in the interaction between the support and the simulation. Arthur Goldberg responded (Sept. 27) that rollback in the Time Warp system would not make debugging a simulation program any harder than debugging it on Misra-type. His approach is to debug the simulation on a centralized simulator, since no matter what system one uses, one needs a centralized simulator with the same interface. The next step is to mu the simulation program on a working Time Warp system. While debugging the simulation on TW, only read committed output, unless you suspect a bug in the Time llarp operating system.

Orna Berry stated that he has been involved in simulations and implementations of the Time 11 ar Mechanism and feels it is rery easy to keep traces of messages (positive and nowative or "messages" and "antimessages") rs. traces of only thene mesages that rere used in the final computation (positive messages that were not caucelfod). Sinaphots can be obtained of erery object, and the event quenes on all machines. so it is not so hard to develop good debugging tools. The University of Calgam has a graphies interface showing important attributes of the objects in the simulation and of the data structures reflecting the simulation time, which makes debugging really pleasant.

The author of this report feels that in relatively simple situations, the Misratype approach is the better one. However, relatively few simulations will be of this type. The Time Warp method is certainly more general, more flexible, and more versatile. One must, however, go to the expense of writing ones own or adapting an existing Time Warp operating system. For a large simulation of the type one would want to perform on a distributed system, that does not seem unreasonable. Attempts to automate Misra-type systems would encounter a similar situation.

Of the other systems that were examined, e.g., the data flow type and the mixed system proposed by Chlamtac, none were considered mature enough in their development to be considered practical at this point. Many left questions as to thejr validity and to how various aspects would be implemented. Indeed, some contained idens that shond be pursued further. 


\subsection{Icleas for Future Research}

One problem with all distributed discrete event simulation methods is that they reguire the somding of many messages that do not correspond to messages in the real systom. In leffurn method antimessages and new messages must be sent when a messige is received that has a timestamp earlier than the local clock time. In Mlismats methor. on must cither avoid deadlock through the use of NLL messages or drice the existence of deadlocts through the use of messages. In any case' the number of extra messages that must be sent becomes large. One area in which research looks frutlul is the area of determining methods by which the sending of at least some of the NULL messages can be avoided.

Some realistic assumptions need to be made on the processing that takes place. One such assumption is that a nonzero minimum time, $T_{\min }$, can be determined for each process such that if $T_{i}$ is the local time of the process, then the timestamp. $T_{0}$, of the next message to be sent by the process is $T_{0} \geq T_{i}+T_{\min }$. The nonzero requirement can be relaxed under certain conditions, but these need to be determined.

The second assumption is that messages are sent when they are ready. The consequence of this assmption is that the receiving process must have sufficient. memory to store all the messages it receires and has not yet processed.

The third assumption is that a process sends messages out on a link in timestamp ordel. It need not. howevel. send messages out in timestamp order along two different paths. Also in keeping with Misia's method. a Logical Process (LP) processes the messages in timestamp order. The LP may have to wait for a message along one or more channels before it can perform processing if messages along those channels could come with a timestamp that is less than that of the messages it has received along the other channels.

Further assume the existence of source processes, that only generate messages; logical processes. that simulate real processes; and sink processes. that only receive messages. Receiving processes know in adrance all of the processes with which it can communicate. A linowledge of this interconnection structure allows a directed graph (digraph) to be constructed.

The digraph can be used for a number of purposes. For example, in the paper by Berry and Jefferson, discussed in Section 2.2.2, a digraph is used to determine a critical path through the distributed processes to determine the amount of parallelism in a distributed program. The idea is to use digraphs to determine cases where NULL messages need not be sent.

First consider a series connection between two processes. The minimum simulation time that must be added to the local time of the first process before the message reaches the last process in the series is the sum of the minimum times of the processes along the path.

Now consider two paths, $A$ and $B$, in parallel from Logical Process $\mathrm{LPa}$ to LPz. Given minimum times for all processes along each path, a minimum time can be precalculated for the simulation time that must be added to the local time of $\mathrm{LPa}$ before reaching $\mathrm{LP} z$ orer each path. If a message is sent by $\mathrm{LPa}$ along path $A$ and 1hat mesage includes the local time of ILPa at the time LPa processed the message. then LI\% can detemine the eallest time at which a message can be received over path 33 . It is the loral time of IJPa plus the minimum time orer path B. 'Therefore a Nl.LL messige need not be passed from LPa to LPz orer" jath B. which would require proccsing by each process along the way.

ln order to take adrantage of the above information. it is helpful to be able 10 determine the structure of the graph. In Section 2.1.6, Yamamoto and 
Hasegawa speak of tree-like loops. However. they do not state how one can find thest. nor are these the only kinds of loop that exist. The digraph can first be simplified by determining all the series connections of processes which can then be represented by a single edge in a new graph that displars the structure of the injtial graph. Series connections do not contribute to the XULL message problem.

A method of determinating loops within the graph is desirable. The concepts of graph decomposition [18] can be used to determine portions of the graph that contain loops. Standard procedures of creating a spanning tree and set of links can be used for this purpose. Tro types of loop can be distinguished, those that can be traversed entirely in the direction of the arrows on the digraph are feedback loops. and those that can be traversed from an LPa to an $\mathrm{LP}_{2}$ along two distinct paths in the direction of the arrows constitutes a feedforward loop.

The most dificult part of the problem is to determine how feedback loops can be handled. In the simple case in which a process receives from two senders. one of which is from a feedluack loop. a minimum time can be calculated for the timestamy of any message that traverses the loop. This knowledge would allow the process to proced in cases where it could not proceed using Misra's rules.

Nonc that the existence of subgraph in series. that is. subgraphs that mav contain loops of some lind but have a single input source and single output channel, implies that NlLL mossiges need not be sent across the boundary between the subgraphs. Onher kinds of infomation should be determinable from the structure of the graph.

Situations exist in which processes can be given information in adrance or can determine information while in operation that would allow the process to make predictions regarding minimum channel times. This information looks particularly useful in allowing processes to get started where they would otherwise be unable to get started using Misra's basic method because of parallel branches or because of loops. The derelopment of methods of exploiting the available information and using graph decomposition appears to be a fruitful area for future research. 


\section{REFERENCES}

[1] K. M. Chandy aud J. Misra, "Deadlock Absence Proofs for Networks of Communicating Processes", Information Processing Letters, Vol. 9, No. 4, Nor. 20. 1979 . pp. 18.5-189.

[2] K. Chandy and J. Misra. "A Case Sudy in Design and Verification of Distributed Progmans. IEEE Transactions on Software Engineering, Vol. SE-5.

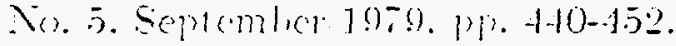

[3! .1. Pracock. 1. M. Ming. and E. Ci. Manning. "Distributed Simulation Lsing

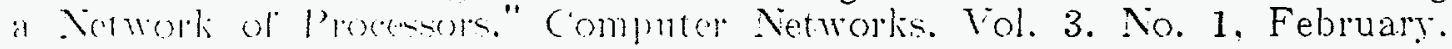
$1079,191 \cdot-1+506$

[-1] K. M. (bandy and J. Misa. "Asychronous Distributed Simulation via a Sequence of farallel Computations." Communications of the ACM, Vol. 24, No. 11, April. 1981, pp. $198-206$.

[5] S. Smoliar, "Simulating Distributed Systens: A Two-Level Approach," Third Annual Conference on Computers in Aerospace, San Diego, CA, October, 2628. $1981, \mathrm{pp} .410-417$.

[6] Y. Yamamoto and T. Hasegawa, "A Time Advancing Algorithm for Distributed Simulation," Modeling And Simulation, Vol 12, Part 2: Systems, Control and Computers; Instrument Society of America; Proceedings of The Twelfth Annual Pittsburgh Conference, April 30-May 1, 1981, pp. 483-493.

[7] K. Chandy, J. Misra, and L. Haas, "Distributed Deadlock Detection," ACM Transactions on Computer Systems, Vol. 1, No. 2, May 1983, pp. 144-156.

[8] T. Yoshida and M. Tokoro, "Distributed Time Management for Discrete System Simulation," Modeling And Simulation, Vol 15, Part 2: Control and Communications: Instrument Society of America: Proceedings of The Fifteenth Annual Pittsburgh Conference, April 19-20. 1984, pp. 751-757.

[9] D. Reed, "Parallel Discrete Event Simulation: A Case Study," 18th Annual Simulation Symposium, Tampa, Florida, Mar. 13-15, 1985 pp. 95-107.

[10] D. Kumar, "Simulating Feedforward Systems Using a Network of Processors," IEEE Computer Society Press; Nineteenth Annual Simulation Symposium, Tampa, Florida, March 12-14, 1986, pp. 127-144.

[11] J. Misra. "Distributed Discrete Event Simulation," Computing Surveys Vol. 18. No. 1. March 1986, pp. 39-65.

[12] R. Bagrodia. K. Chandy, and J. Misra, "A Message-Based Approach to Discrete-Event Simulation," IEEE Transactions on Software Engineering, Vol. SE-13, No. 6, June, 1987, pp. 654-665.

[13] D. R. Jefferson. "Tirmal Time." ACMT Transactions on Programming Languages and syslens. Vol. 7. No. 3. July. 1985. pje f(0-4-425. 
[14\} O. Berry and D. Jeflerson. "Critical Path Analysis of Distributed Simulation," Proceedings of the Conference on Distributed Simulation 1985, P. Reynolds, oditor. Simulation Series. Vol. 15. No. 2. The Society for Computer Simulation. Jan. 2.-20(j. Sin J)iego. California. 1985. pj). 57-60.

[15] J. Sokol. S. Laskorski, and R. Nugent. "Strategies for the Placement of an Object-Oriented Simulation on a Parallel Processor," The Twenty-Second Annual Symposium, Washington Operations Research/Management Science Council, October 16, 1985.

[16] I. Chlamtac. "EDDIE: And Efficient Design for a Distributed Simulation Engine," Proceedings of the Conference on Distributed Simulation 1985, P. Reymolds, editor, Simulation Series, Vol. 15, No. 2, The Society for Computer Sinulation. Jan. 24-26. San Diego, California, 1985, pp. 85-88.

[17] B. Jayaraman, "Dataflow Approach to Discrete Simulation," 1981 Parallel Processing Conference, IEEE, 1981, pp. 158-159.

[18] Deo, Narsingh, Graph Theory with Applications to Engineering and Computer Science, Prentice-Hall, Englewood Cliffs, N.J., 1974. 